\title{
Evaluating quantitative fatty acid signature analysis (QFASA) using harbour seals Phoca vitulina richardsi in captive feeding studies
}

\author{
Chad A. Nordstrom ${ }^{1, *}$, Lindsay J. Wilson ${ }^{1}$, Sara J. Iverson ${ }^{2}$, Dominic J. Tollit ${ }^{1}$ \\ ${ }^{1}$ Marine Mammal Research Unit, Fisheries Centre, Aquatic Ecosystems Research Laboratory, 2202 Main Mall, \\ University of British Columbia, Vancouver, British Columbia V6T 1Z4, Canada \\ ${ }^{2}$ Department of Biology, Dalhousie University, Halifax, Nova Scotia B3H 4J1, Canada
}

\begin{abstract}
Quantitative fatty acid (FA) signature analysis (QFASA) has recently been developed to estimate the species composition of predator diets by statistically comparing FA signatures of predator adipose tissue with that of their potential prey. Captive feeding trials were used to further test the technique with newly weaned harbour seals Phoca vitulina richardsi $(\mathrm{N}=21)$. Two groups of seals were fed monotypic diets of either Pacific herring Clupea pallasii or surf smelt Hypomesus pretiosus for $42 \mathrm{~d}$ while a third group was fed smelt for $21 \mathrm{~d}$ followed by herring for $21 \mathrm{~d}$. Blubber biopsies were taken dorsally at Days 0, 21 and 42. Specific calibration coefficients (CC) used by QFASA were developed from 4 juvenile harbour seals and in some cases differed by 2 -fold with previously reported phocid CC values. The QFASA diet estimates were evaluated using 2 CC sets, 15 FA subsets and a library of 3 to 11 potential prey species. Diet switches were best tracked using the harbour seal CC and the new FA subset. Overall prey misclassifications were apparent (mean $=12 \%$, range $=4$ to $25 \%$ ) when modeled with 8 additional prey not fed, a trend consistent with overlapping prey FA signatures. Blubber FA turnover rates were not strictly linear and in the order of 1.5 to 3 mo in newly weaned seals. Following parameter optimization of the model, QFASA estimates reflected major diet trends in the feeding study, but were sensitive to the CC and FA subsets used as well as to prey species with similar FA signatures. Our results have important implications in the application of QFASA to the study of pinniped diets with more complex feeding histories and wider prey fields.
\end{abstract}

KEY WORDS: Fatty acid $\cdot$ Diet $\cdot$ Blubber $\cdot$ Harbour seal $\cdot$ Phoca vitulina $\cdot$ QFASA

\section{INTRODUCTION}

Determining the diets of predators is of primary importance to assess their role in an ecosystem. Identifying and quantifying which prey species are consumed is made difficult when foraging takes place in an aquatic environment. Traditional methods of diet reconstruction include the identification of prey remains recovered from the stomach contents of harvested animals or from scat samples collected at haulouts (areas where pinnipeds congregate on land). These methods are subject to well-documented biases, as prey may not be wholly consumed, identifiable structures may be lost or may degrade at species-specific rates and soft-bodied prey may be digested com- pletely (Jobling \& Breiby 1986, Pierce \& Boyle 1991, Staniland 2002). There is also debate as to how to enumerate recovered hard parts and subsequently reconstruct past diet (Laake et al. 2002, Tollit et al. 2003, Trites \& Joy 2005). In addition, samples from these methods represent feeding events from the previous few days, limiting the time scale for which the data are relevant.

The use of signatures of fatty acid(s) (FA) is a relatively new method to evaluate diets of predators at the top of the food web based on the demonstrated influence of dietary FA on predator fat stores (Rouvinen \& Kiiskinen 1989, Colby et al. 1993, Raclot et al. 1998). Diet is the primary source of many FA for most monogastric animals, which are subsequently incorporated 
into adipose tissue as triacylglycerols. A relatively small number of FA can be synthesized de novo in higher predators (e.g. fish, birds and mammals), but long-chain FAs, particularly omega-6 and omega-3 FAs, cannot be endogenously produced. Marine food webs contain many long-chain and unsaturated FA (e.g. greater than C18:0) that are believed to be specific to individual prey species and ecosystem regions (e.g. Budge et al. 2002, Iverson et al. 2002). The premise of using FA signatures to estimate diet is based on the principle that identifiable long-chain FA from prey species are incorporated into the blubber of the predator with minimal modification and/or in a predictable fashion (Iverson 1993). It has been postulated that one can make predictions concerning dietary history by sampling the metabolically active fat layer of the predator, identifying key diet-related FA concentrations and then comparing them with the FA concentrations of potential prey. Using FA signatures to describe diet has the advantages of potentially identifying important dietary components missed by using stomach content or scat analyses and, as a storage tissue, blubber FA represent diet over a longer (yet uncertain) feeding period.

Fatty acid techniques have been used qualitatively to indicate temporal or geographical variation in diet for a variety of cetaceans (Dahl et al. 2000, Hooker et al. 2001, Samuel \& Worthy 2004, Herman et al. 2005, Ruchonnet et al. 2006), pinnipeds (Smith et al. 1996, Iverson et al. 1997, Lea et al. 2002, Walton \& Pomeroy 2003, Andersen et al. 2004, Arnould et al. 2005, GrahlNielsen et al. 2005) and the polar bear Ursus maritimus (Grahl-Nielsen et al. 2003, Thiemann et al. 2006). While a predator's metabolism precludes its own FA signature from exactly matching that of its prey (Iverson et al. 2004), some investigators have linked predator and prey FA using various qualitative techniques (e.g. Iverson et al. 1997, Kirsch et al. 2000, Bradshaw et al. 2003).

Quantitative fatty acid signature analysis (QFASA) is a multivariate optimization model designed to predict the relative proportion of prey species in the diet of marine predators (Iverson et al. 2004, 2006, in press, Budge et al. 2006, Beck et al. 2007). The analysis requires a library of FA profiles and lipid contents from potential prey to compare with the predators' blubber FA profile as well as calibration coefficients (CCs) to correct for effects of biosynthesis or modification of specific FA in the predator. To date, efforts to establish CCs and test the efficacy and interpretation of QFASA have been confined to a few species (Iverson et al. 2004, 2006, in press, Hoberecht 2006, Tollit et al. 2006). Diet estimates generated from these studies have varied, sometimes significantly, depending on the CCs and/or the FA subset employed, even when only prey actually fed was used as input. Assessment of model accuracy and prey misclassification rates (i.e. occasions when the model may predict a prey species was consumed when it was not or when consumed prey was not detected) has been largely confined to computer simulations using 'pseudopredators.' Difficulties have been noted in distinguishing prey with similar FA signatures, but on average absolute error rates have been low (e.g. $<10 \%$, range $0-40 \%$ ). These studies have stressed the need for further testing of the QFASA model with additional controlled feeding studies to assess FA signature deposition, test the influence of FA subsets and estimate blubber FA turnover rates (i.e. period of dietary history). Incorporating prey that have not been fed in the model would also be important when attempting to replicate conditions encountered when analyzing samples collected from freeranging animals. Our goal was to evaluate QFASA via captive feeding trials using 21 harbour seals Phoca vitulina richardsi to: (1) qualitatively separate prey samples by species and blubber samples by feeding regime, (2) obtain species-specific CCs, (3) optimize appropriate CC and FA subsets for use when estimating diet, (4) compare the accuracy of QFASA model outputs with known dietary histories, and (5) estimate blubber turnover time in newly weaned seals.

\section{MATERIALS AND METHODS}

Feeding study. The feeding study took place between August 28 and October 9, 2003 with 21 harbour seals housed at the Vancouver Aquarium Marine Mammal Rescue facility (Table 1). All seals brought to the facility were estimated to be $<1$ mo old; there was no data on their feeding history. They were housed in individual tubs and were tube-fed a homogenous mixture consisting of 2 parts herring to 1 part salmon oil (by weight) for approximately $3 \mathrm{wk}$. Seals were then 'weaned' onto whole herring over 4 to $6 \mathrm{~d}$ after which they received solely herring until the start of the experimental period (Table 1). Seals were transferred to larger shared pools as they increased in size, during which time every effort was made to feed seals individually.

'Weaned' seals were placed in 1 of 3 diet treatments for a $42 \mathrm{~d}$ controlled feeding period. Two groups of seals were fed monotypic diets of either Pacific herring Clupea pallasii or surf smelt Hypomesus pretiosus for $42 \mathrm{~d}$ while a third group was fed smelt for $21 \mathrm{~d}$ followed by herring for $21 \mathrm{~d}$ (Fig. 1). Seals were typically fed 10\% (herring diet) or $20 \%$ (smelt diet) of their body mass daily resulting in similar percentage body mass increases across the study (Table 1). Blubber biopsies were taken at Day 0 (termed Biopsy 'A'), Day 21 (Biopsy 'B') and Day 42 (Biopsy ' $\mathrm{C}$ '). In addition, a single full 
Table 1. Phoca vitulina. Sex ( $\mathrm{M}=$ male, $\mathrm{F}=$ female), treatment group, number of days at the facility before 1st biopsy (parentheses: number of days fed only herring) and mass at time of sampling of 21 newly weaned harbour seals in the feeding study.

${ }^{*}$ Feeding regime not completed and biopsies B and C excluded from further analysis

\begin{tabular}{|c|c|c|c|c|c|c|}
\hline Animal no. & Sex & Feeding group & $\begin{array}{l}\text { Days before 1st biopsy } \\
\text { (days fed only herring) }\end{array}$ & Day 0, Biopsy A & $\begin{array}{l}\text { Mass }(\mathrm{kg})- \\
\text { Day 21, Biopsy B }\end{array}$ & Day 42, Biopsy C \\
\hline Pv03-01 & $\mathrm{M}$ & Herring Only & $52(26)$ & 12.2 & 20.4 & 23.2 \\
\hline Pv03-02 & M & Herring Only & $38(22)$ & 14.6 & 20.0 & 22.6 \\
\hline Pv03-06 & M & Herring Only & $47(30)$ & 19.8 & 16.6 & 20.0 \\
\hline Pv03-07 & M & Herring Only & $53(30)$ & 24.6 & 32.4 & N/A \\
\hline Pv03-08 & M & Herring Only & $33(17)$ & 16.4 & 26.4 & N/A \\
\hline Pv03-09 & $\mathrm{F}$ & Herring Only & $51(30)$ & 20.0 & 28.6 & N/A \\
\hline Pv03-11 & $\mathrm{F}$ & Herring Only & $33(18)$ & $\begin{array}{c}13.0 \\
\text { Mean }=17.2\end{array}$ & $\begin{array}{c}20.8 \\
\text { Mean }=23.6\end{array}$ & $\begin{array}{c}\text { N/A } \\
\text { Mean }=21.9\end{array}$ \\
\hline Pv03-04 & $\mathrm{M}$ & Smelt Only & $36(17)$ & 13.2 & 15.6 & 17.4 \\
\hline Pv03-05 & M & Smelt Only & $37(18)$ & 10.8 & 14.0 & 16.6 \\
\hline Pv03-12 & M & Smelt Only & 31 (12) & 12.2 & 16.6 & 20.0 \\
\hline Pv03-13 & $\mathrm{F}$ & Smelt Only & $34(18)$ & 13.6 & 17.8 & 18.6 \\
\hline Pv03-16 & $\mathrm{M}$ & Smelt Only & $23(12)$ & 11.8 & 16.0 & 17.6 \\
\hline Pv03-17 & $\mathrm{M}$ & Smelt Only & $23(04)$ & 10.8 & 14.0 & 15.4 \\
\hline Pv03-20 & M & Smelt Only & $21(04)$ & $\begin{array}{c}11.8 \\
\text { Mean }=12.0\end{array}$ & $\begin{array}{c}16.2 \\
\text { Mean }=15.7\end{array}$ & $\begin{array}{c}20.6 \\
\text { Mean }=18.0\end{array}$ \\
\hline Pv03-03 & $\mathrm{M}$ & Smelt-Herring & $40(18)$ & 14.4 & 14.8 & 21.8 \\
\hline Pv03-10 & $\mathrm{F}$ & Smelt-Herring & 47 (18) & 10.0 & 10.4 & 15.2 \\
\hline Pv03-15 & $\mathrm{F}$ & Smelt-Herring & $24(04)$ & 9.0 & 9.8 & 15.2 \\
\hline Pv03-18 & $\mathrm{M}$ & Smelt-Herring & $23(04)$ & 10.0 & 12.6 & 18.2 \\
\hline Pv03-19 & $\mathrm{F}$ & Smelt-Herring & $21(04)$ & 9.4 & 10.6 & 17.0 \\
\hline Pv03-21 & $\mathrm{F}$ & Smelt-Herring & $50(11)$ & 12.6 & 15.8 & 22.6 \\
\hline Pv03-14 & $\mathrm{M}$ & ${ }^{*}$ Smelt-Herring & $26(12)$ & $\begin{array}{c}9.4 \\
\text { Mean }=10.7\end{array}$ & $\begin{array}{c}9.2 \\
\text { Mean }=11.9\end{array}$ & $\begin{array}{c}13.0 \\
\text { Mean }=17.6\end{array}$ \\
\hline
\end{tabular}

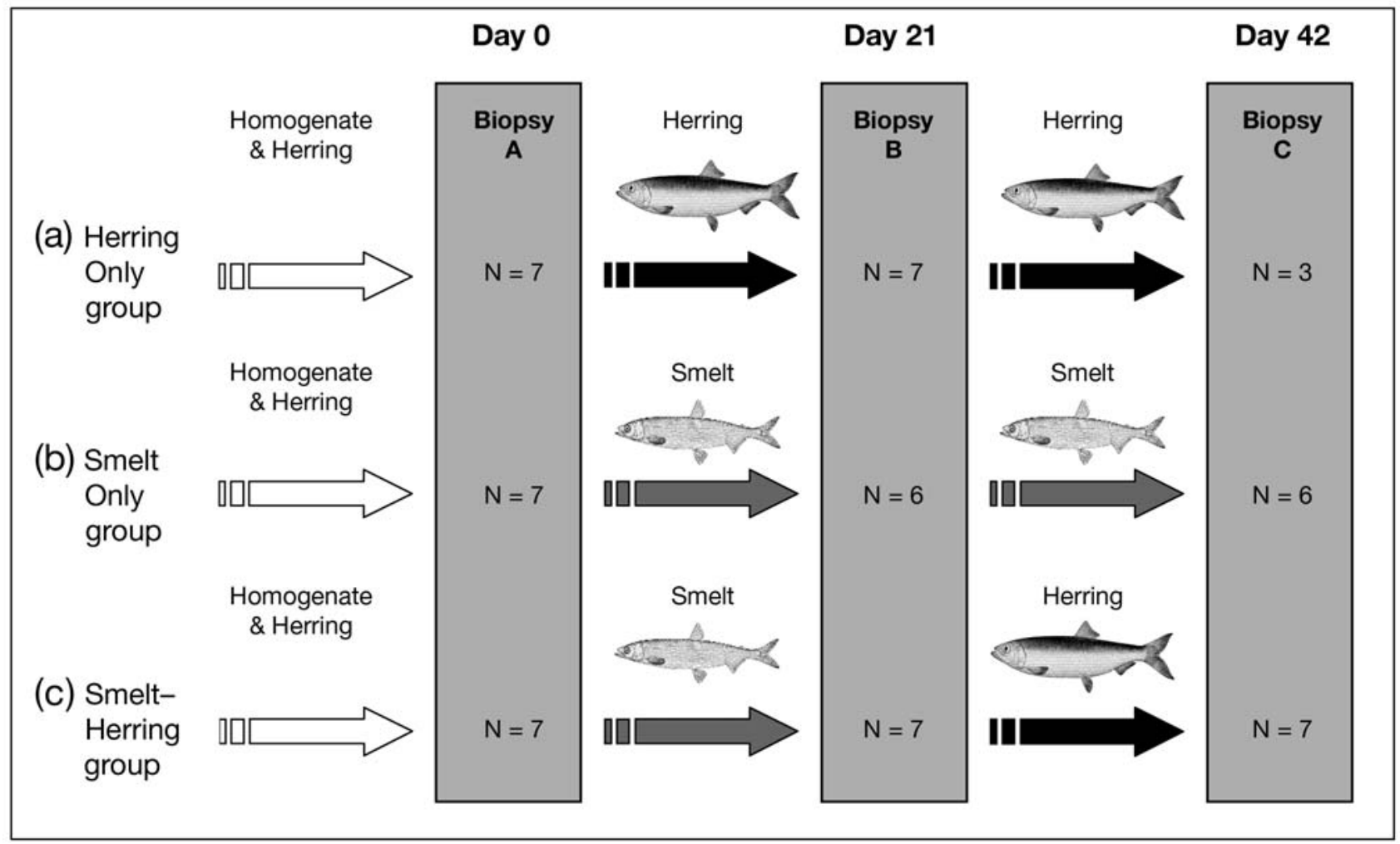

Fig. 1. Phoca vitulina. Feeding regimes for (a) Herring Only, (b) Smelt Only, and (c) Smelt-Herring treatment groups over 42 d. Number of seals $(\mathrm{N})$ sampled at each biopsy noted for each group. Arrows indicate diet fed (white = homogenate and herring, black $=$ herring, grey $=$ smelt) 
depth blubber biopsy was taken from captive juvenile harbour seals $(\mathrm{N}=4)$ housed at the Vancouver Aquarium to obtain species-specific CCs. These juveniles had eaten herring from a single lot exclusively for $>1 \mathrm{yr}$ prior to their biopsies and herring $(\mathrm{N}=23)$ were subsampled for FA analysis during this period. Smelt fed in the study comprised gravid and non-gravid fish as well as a smaller, non-gravid, size class. Sub-samples were taken from each of the 3 smelt types ( $N=40$ total, Table 2). Herring and salmon oil homogenates were from a single lot of herring and salmon oil, respectively. On average 30 prey items from 8 other species were also sub-sampled for FA analysis (Table 2).

Biopsy sampling. Animal restraint and biopsy sampling followed guidelines of the University of British Columbia animal care committee and was carried out under authorization by the Vancouver Aquarium research board. Seals were physically restrained on a purpose-built restraint board. The area on the midflank, considered the area of greatest fat depth $(5 \mathrm{~cm}$ above the pelvic girdle and $2 \mathrm{~cm}$ from the edge of the spinal column), was shaved and sanitized with isopropyl alcohol and iodine before $1.5 \mathrm{ml}$ of local anaesthetic (Lidocaine, $20 \mathrm{mg} \mathrm{ml}^{-1}$ ) was injected at 4 positions around the site. A veterinarian made a $2 \mathrm{~cm}$ incision on the surface of the skin to allow for the insertion of a $6 \mathrm{~mm}$ biopsy punch. The biopsy punch was turned, with gentle downward pressure, to form a plug in the blubber to the level of the fascia while aiming to avoid penetration into the muscle (i.e. the entire depth of the blubber layer was sampled) (Thiemann et al. 2004). The blubber core was removed with forceps or angled scissors and immediately wrapped in aluminium foil. The area received a topical antibiotic and seals were placed on a $3 \mathrm{~d}$ course of oral antibiotics as a precautionary measure. All biopsy sites healed within 2 to $3 \mathrm{wk}$ and no infections requiring further treatment occurred. Sampling methods were identical for all seals throughout the study, with successive samples alternating between flanks. The 4 larger juvenile seals also received an additional intramuscular analgesic 20 min before the biopsy procedure (Butorphanol tartrate, $10 \mathrm{mg} \mathrm{ml}^{-1}$ solution applied at $0.02 \mathrm{ml} \mathrm{kg}^{-1}$ ). Samples were divided in half to potentially assess FA stratification (inner versus outer layers); however, results from the whole biopsy are presented here to focus the study on what is considered the optimal sample (see Budge et al. 2006). Samples were immediately weighed, placed in a solution of chloroform and $0.01 \%$ butylated hydroxytoluene (BHT) and stored at $-30^{\circ} \mathrm{C}$ until FA extraction and analysis.

FA identification. Lipids were extracted from homogenized harbour seal blubber and whole prey samples using a modified Folch technique (Folch et al. 1957, Iverson et al. 2001). The relative contribution of 68 FA was determined using flame ionization detector gas layer chromatography for each sample as described in Budge et al. (2002). Detailed specifics of the laboratory analyses can be obtained from Iverson (1993) and Iverson et al. $(1997,2004)$. All blubber FA composition analyses were undertaken at the Department of Biology, Dalhousie University, without prior knowledge of specific feeding regime.

Qualitative separation of prey and blubber FA. Qualitative analyses were conducted using a backward stepwise discriminant function analysis (DFA) (alpha values 0.15, Systat, v.11) to assess the extent traditional statistical tests could separate prey groupings by species or animal groups by diet and biopsy date. The 3 types of smelt were considered as different prey groupings in the analyses. To minimize overparameterization we selected 9 prey FA which had the

Table 2. Mean \pm SD morphometric data and lipid content for 307 prey species included in the QFASA prey library. Surf smelt prey are sub-categorized by size (large, small) and sexual state (gravid, non-gravid). na = not available

\begin{tabular}{|c|c|c|c|c|c|}
\hline Prey species & Scientific name & $\mathrm{N}$ & Length $(\mathrm{cm})$ & Mass (g) & Lipid (wet wt \%) \\
\hline Pacific herring & Clupea pallasii & 23 & $18.8 \pm 1.64$ & $87.6 \pm 19.75$ & $11.2 \pm 1.95$ \\
\hline $\begin{array}{l}\text { Herring and } \\
\text { salmon oil homogenate }\end{array}$ & na & 5 & na & na & $23.6 \pm 2.95$ \\
\hline Surf smelt (large, gravid) & Hypomesus pretiosus & 10 & $14.7 \pm 0.59$ & $38.1 \pm 6.07$ & $3.9 \pm 2.34$ \\
\hline Surf smelt (large, non-gravid) & Hypomesus pretiosus & 20 & $14.9 \pm 0.67$ & $42.0 \pm 7.54$ & $2.3 \pm 0.98$ \\
\hline Surf smelt (small, non-gravid) & Hypomesus pretiosus & 10 & $10.6 \pm 0.56$ & $12.5 \pm 1.75$ & $2.2 \pm 1.45$ \\
\hline Atka mackerel & Pleurogrammus monopterygius & 24 & $33.2 \pm 1.65$ & $463.9 \pm 60.32$ & $5.9 \pm 2.79$ \\
\hline Capelin & Mallotus villosus & 54 & $14.2 \pm 0.80$ & $25.9 \pm 4.38$ & $3.3 \pm 1.21$ \\
\hline Coho salmon & Oncorhynchus kisutch & 38 & $28.3 \pm 2.97$ & $358.7 \pm 98.56$ & $3.8 \pm 1.09$ \\
\hline Eulachon & Thaleichthys pacificus & 30 & $17.4 \pm 1.56$ & $47.4 \pm 11.79$ & $8.8 \pm 2.84$ \\
\hline Pilchard & Sardinops sagax & 18 & $23.6 \pm 1.49$ & $202.0 \pm 34.84$ & $20.3 \pm 4.79$ \\
\hline Walleye pollock & Theragra chalcogramma & 17 & $34.7 \pm 1.45$ & $391.6 \pm 35.20$ & $6.9 \pm 1.10$ \\
\hline Pacific sandlance & Ammodytes hexapterus & 15 & $9.9 \pm 2.00$ & $4.3 \pm 2.72$ & $5.7 \pm 1.76$ \\
\hline California market squid & Loligo opalescens & 43 & na & $35.1 \pm 8.69$ & $2.2 \pm 0.36$ \\
\hline
\end{tabular}


highest variance and an overall mean of $>0.25 \%$ of the 68 total FA $(16: 4 n-1,18: 1 n-9,20: 1 n-7,20: 1 n-9$, 20:1n-11, 20:4n-6, 22:1n-11, 22:1n-9, 22:6n-3), and 6 blubber dietary FA with the highest observed variance across animal groupings $(16: 0,16: 1 \mathrm{n}-7,18: 1 \mathrm{n}-9$, 20:1n-9, 20:5n-3, 22:6n-3). Percentage values for FA were renormalized to $100 \%$ and arcsine transformed prior to analysis.

CC and FA subset model optimization. New CC values were calculated by dividing FA levels in the 4 juvenile harbour seals by FA levels in the 23 herring resulting in 92 values per FA. Each set of 92 FA values was then ordered and the 3 highest and lowest values removed (termed '10\% trim') before calculating a mean value for each FA (sensu Iverson et al. 2004). Given that FA metabolism may vary during early development and weaning, diet estimates were generated using CCs based on our juvenile harbour seals and also using CCs previously determined for grey seal Halichoerus grypus pups (reviewed in Iverson et al. 2004) for comparison. Individual blubber samples from the feeding study were calibrated using each CC set separately and performance was gauged for the 15 FA subsets tested (see next).

Fifteen subsets of the 68 FA identified were tested in our model optimization exercise (see Table 4, Appendix 1). Each subset was composed of 33 to 41 individual FA and subsets were re-normalized to $100 \%$ before each evaluation. Thirteen new FA subsets were developed based on 2 published subsets that include FA that arise from dietary origin alone (Dietary, 33 FAs) or that encompass the Dietary and 8 other FA influenced by the consumption of specific prey (Extended Dietary, 41 FAs) (Iverson et al. 2004). Select FA were omitted in the new subsets mainly due to high levels of variability observed in the CCs. Subset optimization was based on prey misclassification levels using the full prey library (11 species) and using a pre-validated version of the QFASA model (FasCalc v. 1.0, M. J. Walton, Sea Mammal Research Unit, University of St. Andrews). Mean prey species signatures were used (i.e. no resampling of individual prey items). New subset performance was initially screened using 3 biopsies (Biopsy A, Biopsy B and Biopsy C) from 2 seals from each treatment group ( $\mathrm{N}=6$ seals, 18 biopsies total). The new FA subset that provided the lowest estimate of unfed prey (termed misclassified species) was subsequently compared with the published Dietary and Extended Dietary subsets using all experimental biopsies.

QFASA model evaluation post optimization. After defining our optimal CC and FA subset, each calibrated blubber biopsy was modelled separately to assess the ability of QFASA to identify only prey fed using 3 variations of the prey library. The full 11-species prey library included the 3 known prey fed (her- ring, smelt and homogenate) and 8 other potential prey species, aiming to mimic an optimal situation in the wild where a limited number of prey are available to the predator. The 10 species library excluded coho salmon Oncorhynchus kisutch as a result of overlap observed between smelt and coho salmon in the prey DFA analysis (see Results). Finally, only the 3 known fed prey species were modelled. The 3 smelt classes previously described were modelled as separate prey and the results pooled to provide an overall smelt proportion. The QFASA diet estimates (as wet weight percent) for homogenate, herring, smelt and other prey (i.e. misclassified) species were averaged for each treatment group and misclassifications were noted. Confidence intervals were calculated following arcsine transformation, but have been back-transformed and presented in original units (Zar 1996).

Statistical differences across biopsies (within each diet group) and across diets (within each biopsy period) were examined for each variation of the prey library using arcsine transformed herring and smelt QFASA estimates in a linear mixed effects model. In addition, we directly compared the absolute variance of QFASA diet estimates (Biopsy C, 3-species prey library) on an individual animal basis with the cumulative recorded percent mass intake at 40,55 and $65 \mathrm{~d}$ before Biopsy C. A deviance value of 0 indicated an even match between the QFASA estimated diet and the cumulative recorded diet over the assessment period.

Blubber FA signature turnover. We employed 2 methods to assess FA turnover: (1) an approach that used the rate of increase in the proportion of smelt estimated by QFASA over the experiment for the smelt fed seals, and (2) chi-square statistical comparisons of QFASA herring and smelt estimates with cumulative recorded diet at different temporal scales. Daily feeding records were used to calculate the mean proportion of homogenate, herring and smelt (by weight) consumed by each group at $5 \mathrm{~d}$ intervals between 35 and $75 \mathrm{~d}$ before each biopsy (whenever possible). Blubber samples were modelled with optimal parameterization using the harbour seal CC set, the optimal FA subset (Reduced) and the 3-species prey library (known prey fed only) for both methods.

\section{RESULTS}

\section{Biopsy sampling and FA summary}

A total of 63 harbour seal biopsies were collected (59 from newly weaned seals, 4 from calibration seals). Not all 21 seals were able to complete the feeding study, resulting in unequal sample sizes across diet groups 
(Table 1). Four seals (Pv03-07, Pv03-08, Pv03-09, Pv0311) from the Herring Only group were released $13 \mathrm{~d}$ after Biopsy B after completing the rescue program and were, therefore, not available for a third biopsy.

Also, animal Pv03-14 from the Smelt-Herring group abruptly ceased eating the offered smelt and was returned to a herring diet $11 \mathrm{~d}$ earlier than planned. Samples obtained from this animal at Biopsy B and Biopsy C were subsequently removed from the analyses. Notably, seals in this group consistently showed the least mass gains from Biopsy A to Biopsy B (Table 1).

Whole blubber FA signatures were dominated by monounsaturated FA (MUFAs) in all harbour seals, but the proportions of individual FA varied among diet groupings and the 4 calibration seals (Table 3 ). The calibration seals had lower proportions of all 4 saturated FA (SFAs, particularly 14:0 and 16:0), as well as $22: 1 \mathrm{n}-9,18: 4 \mathrm{n}-3$ and $20: 5 \mathrm{n}-3$, but a far higher proportion of the MUFA 18:1n-9 compared with the experimental diet groups. The SFAs 15:0 and 18:0 increased in blubber after $42 \mathrm{~d}$ of smelt diet, while conversely 14:0 and 16:0 were lowest after $42 \mathrm{~d}$ of smelt. The unsaturated FAs, 14:1n-5, 16:1n-7, 20:1n-7, 20:4n-6 and 22:6n-3, were all also relatively high after a diet of smelt compared with a diet of herring, while some of the longer chain FAs, notably 20:1n-9, 20:1n-11, 22:1n-9 and 22:1n-11, were relatively low (Table 3 ).

The 15 most abundant FA in the 307 individual prey typically included 14:0, 16:0, 16:1n-7, 18:0, 18:1n-7, 18:1n-9, 18:2n-6, 18:4n-3, 20:1n-11, $20: 1 \mathrm{n}-9, \quad 20: 4 \mathrm{n}-6, \quad 20: 5 \mathrm{n}-3, \quad 22: 1 \mathrm{n}-11$, 22:5n-3 and 22:6n-3, which together accounted for $88.9 \%$ of the total FAs. Although the most abundant FA were generally similar across prey groupings, the levels varied greatly among species (prey FA data are available from the authors by request). For example, the lowest levels of $16: 1 \mathrm{n}-7$ were consistently found in California market squid Loligo opalescens $($ mean $=0.8 \%$ ), while the highest levels were found in capelin Mallotus villosus $(8.1 \%)$ and pilchard Sardinops sagax $(7.4 \%) ; 22: 1 \mathrm{n}-11$ was low $(<1 \%)$ in squid, Pacific sandlance Ammodytes hexapterus, surf smelt, coho salmon and eulachon Thaleichthys pacificus, but high (10.9\% to $15.3 \%$ ) in capelin, walleye pollock Theragra chalcogramma and Atka mackerel Pleurogrammus monopterygius. Squid had very high levels (34.1\%) of $22: 6 n-3$, but this FA was far less abundant in eulachon $(4.8 \%)$ and herring $(7.6 \%)$.

\section{Qualitative separation of prey and blubber samples}

Prey and blubber samples were differentiated by select FA using DFA. The 9 FA selected for the prey DFA represented $41.6 \%$ of the total FA identified in all prey. Discriminant function (DF) 1 was primarily defined by 20:1n-7, 20:1n-9 and 20:4n-6, while DF 2 was mainly defined by $20: 1 \mathrm{n}-7,20: 1 \mathrm{n}-11$ and $22: 1 \mathrm{n}-11$ (Wilks' lambda, $\mathrm{p}<0.0001$; Fig. 2). DFs 1 and 2 accounted for $58.6 \%$ of the total variance. Prey were correctly classified to species with 297 of 307 (97\%) of original grouped cases and with $96 \%$ of crossvalidated grouped cases. Individual prey grouping classifications exceeded $92 \%$ correct with the exception of the small smelt class, for which 4 of 10 fish were classified as coho salmon. One of 23 herring was also classified as coho salmon.

Significant differences existed between animal groupings for all 6 FA selected for the predator blubber DFA (Wilks' lambda, p < 0.0001; Fig. 3). DF 1 was primarily defined by $16: 0$ and 22:6n-3, while DF 2 was mainly defined by $16: 1 \mathrm{n}-7$. DFs 1 and 2 accounted for $91.7 \%$ of the total variance. Biopsy groupings were separated with $85 \%$ of original grouped cases and with $80 \%$ of cross-validated grouped cases classified correctly. All 4 calibration samples were correctly categorized, as were all but one Smelt Only Biopsy C sample, which was incorrectly classified as a Smelt Biopsy B sample. The poorest classification was of samples after $21 \mathrm{~d}$ of herring (Biopsy B), of which 2 were indistin-

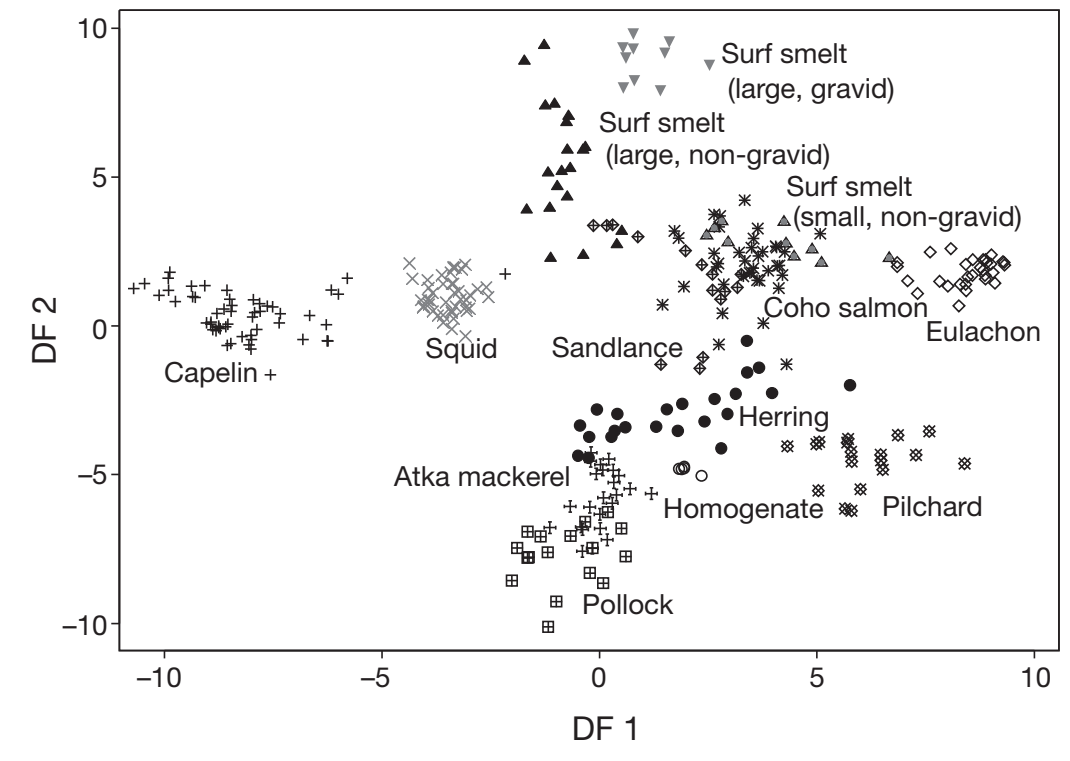

Fig. 2. Discriminant function analysis (DFA) plot of prey samples (see Table 2 for taxonomic names of species) based on 9 FA (16:4n-1, 18:1n-9, 20:1n-7, 20:1n-9, 20:1n-11, 20:4n-6, 22:1n-11，22:1n-9，22:6n-3). Discriminant functions (DFs) 1 and 2 accounted for $58.6 \%$ of the total variance. Prey items were correctly classified to species with $97 \%$ of original grouped cases and $96 \%$ of crossvalidated grouped cases 
Table 3. Phoca vitulina. Mean \pm SD concentrations (weight $\%$ ) of fatty acid (FA) that averaged $>0.20$ of the percentage of total FA from whole blubber biopsies of long-term herring-fed seals used for calibration coefficient estimates or from study seals. ${ }^{*} \mathrm{FAs}$ used in blubber discriminant function analysis. SAT = saturated FA, MUFA = monounsaturated FA, PUFA = polyunsaturated FA

\begin{tabular}{|c|c|c|c|c|c|c|c|}
\hline Fatty acid & $\begin{array}{c}\text { Calibration } \\
\text { coefficient } \\
\text { seals } \\
(\mathrm{N}=4)\end{array}$ & $\begin{array}{c}\text { Homogenate/ } \\
\text { herring } \\
\text { Day 0 } \\
\text { Biopsy A } \\
(\mathrm{N}=21)\end{array}$ & $\begin{array}{c}\text { Herring Only } \\
\text { treatment } \\
\text { Day } 21 \\
\text { Biopsy B } \\
(\mathrm{N}=7)\end{array}$ & $\begin{array}{c}\text { Herring Only } \\
\text { treatment } \\
\text { Day } 42 \\
\text { Biopsy C } \\
(\mathrm{N}=3)\end{array}$ & $\begin{array}{c}\text { Smelt } \\
\text { treatment } \\
\text { Day } 21 \\
\text { Biopsy B } \\
(\mathrm{N}=13)\end{array}$ & $\begin{array}{c}\text { Smelt Only } \\
\text { treatment } \\
\text { Day } 42 \\
\text { Biopsy C } \\
(\mathrm{N}=7)\end{array}$ & $\begin{array}{c}\text { Smelt-Herring } \\
\text { treatment } \\
\text { Day } 42 \\
\text { Biopsy C } \\
(\mathrm{N}=6)\end{array}$ \\
\hline \multicolumn{8}{|l|}{ SAT } \\
\hline 14:0 & $2.87 \pm 0.14$ & $5.12 \pm 0.19$ & $5.13 \pm 0.19$ & $4.51 \pm 0.07$ & $4.60 \pm 0.25$ & $4.35 \pm 0.11$ & $4.51 \pm 0.10$ \\
\hline $15: 0$ & $0.21 \pm 0.00$ & $0.31 \pm 0.04$ & $0.32 \pm 0.01$ & $0.28 \pm 0.01$ & $0.33 \pm 0.04$ & $0.41 \pm 0.02$ & $0.30 \pm 0.01$ \\
\hline $16: 0^{*}$ & $9.05 \pm 0.59$ & $13.22 \pm 0.98$ & $13.23 \pm 0.74$ & $12.51 \pm 0.37$ & $12.09 \pm 1.08$ & $11.49 \pm 0.40$ & $12.29 \pm 0.74$ \\
\hline $18: 0$ & $1.02 \pm 0.12$ & $1.16 \pm 0.19$ & $1.09 \pm 0.07$ & $1.13 \pm 0.08$ & $1.25 \pm 0.19$ & $1.35 \pm 0.05$ & $1.15 \pm 0.12$ \\
\hline \multicolumn{8}{|l|}{ MUFA } \\
\hline $14: 1 n-5$ & $0.94 \pm 0.10$ & $1.84 \pm 1.01$ & $1.00 \pm 0.16$ & $0.87 \pm 0.15$ & $1.85 \pm 0.76$ & $1.47 \pm 0.16$ & $1.06 \pm 0.32$ \\
\hline $16: 1 \mathrm{n}-7^{*}$ & $15.86 \pm 0.99$ & $20.64 \pm 5.61$ & $16.46 \pm 0.74$ & $15.02 \pm 1.07$ & $20.91 \pm 4.63$ & $19.38 \pm 1.21$ & $16.23 \pm 2.33$ \\
\hline $16: 1 n-9$ & $0.47 \pm 0.03$ & $0.32 \pm 0.07$ & $0.28 \pm 0.04$ & $0.28 \pm 0.01$ & $0.41 \pm 0.10$ & $0.43 \pm 0.06$ & $0.32 \pm 0.06$ \\
\hline $16: 1 \mathrm{n}-11$ & $0.68 \pm 0.07$ & $0.49 \pm 0.09$ & $0.59 \pm 0.03$ & $0.57 \pm 0.06$ & $0.53 \pm 0.06$ & $0.59 \pm 0.02$ & $0.58 \pm 0.05$ \\
\hline $17: 1$ & $0.40 \pm 0.01$ & $0.30 \pm 0.05$ & $0.31 \pm 0.01$ & $0.31 \pm 0.00$ & $0.39 \pm 0.04$ & $0.48 \pm 0.02$ & $0.33 \pm 0.02$ \\
\hline $18: 1 n-5$ & $0.22 \pm 0.01$ & $0.34 \pm 0.04$ & $0.30 \pm 0.01$ & $0.29 \pm 0.01$ & $0.30 \pm 0.07$ & $0.27 \pm 0.02$ & $0.31 \pm 0.03$ \\
\hline $18: 1 n-7$ & $3.99 \pm 0.18$ & $3.90 \pm 0.54$ & $4.13 \pm 0.07$ & $4.30 \pm 0.01$ & $3.98 \pm 0.70$ & $4.41 \pm 0.18$ & $4.27 \pm 0.08$ \\
\hline $18: 1 n-9^{*}$ & $32.45 \pm 1.45$ & $20.58 \pm 1.79$ & $22.43 \pm 0.92$ & $22.39 \pm 0.48$ & $19.34 \pm 2.07$ & $19.04 \pm 0.79$ & $20.87 \pm 1.00$ \\
\hline $18: 1 n-11$ & $1.46 \pm 0.13$ & $0.60 \pm 0.13$ & $0.58 \pm 0.06$ & $1.31 \pm 0.23$ & $0.79 \pm 0.27$ & $0.70 \pm 0.15$ & $1.28 \pm 0.15$ \\
\hline $20: 1 n-7$ & $0.17 \pm 0.01$ & $0.20 \pm 0.03$ & $0.20 \pm 0.01$ & $0.22 \pm 0.01$ & $0.35 \pm 0.07$ & $0.50 \pm 0.50$ & $0.26 \pm 0.01$ \\
\hline $20: 1 n-9^{*}$ & $4.00 \pm 0.30$ & $3.43 \pm 0.77$ & $4.04 \pm 0.14$ & $4.51 \pm 0.16$ & $2.55 \pm 0.95$ & $1.80 \pm 0.40$ & $4.09 \pm 0.61$ \\
\hline $20: 1 n-11$ & $2.33 \pm 0.29$ & $2.40 \pm 0.64$ & $2.11 \pm 0.19$ & $2.21 \pm 0.15$ & $1.89 \pm 0.66$ & $1.19 \pm 0.22$ & $2.08 \pm 0.39$ \\
\hline $22: 1 n-9$ & $0.11 \pm 0.01$ & $0.20 \pm 0.04$ & $0.21 \pm 0.02$ & $0.23 \pm 0.02$ & $0.22 \pm 0.05$ & $0.19 \pm 0.03$ & $0.24 \pm 0.03$ \\
\hline $22: 1 n-11$ & $1.11 \pm 0.22$ & $2.37 \pm 0.63$ & $2.77 \pm 0.42$ & $2.96 \pm 0.41$ & $1.53 \pm 0.78$ & $0.86 \pm 0.30$ & $2.81 \pm 0.58$ \\
\hline \multicolumn{8}{|l|}{ PUFA } \\
\hline $16: 3 n-4$ & $0.29 \pm 0.04$ & $0.37 \pm 0.09$ & $0.47 \pm 0.01$ & $0.44 \pm 0.01$ & $0.28 \pm 0.07$ & $0.25 \pm 0.03$ & $0.40 \pm 0.04$ \\
\hline $16: 3 n-6$ & $0.56 \pm 0.08$ & $0.60 \pm 0.12$ & $0.74 \pm 0.01$ & $0.70 \pm 0.03$ & $0.48 \pm 0.10$ & $0.41 \pm 0.05$ & $0.65 \pm 0.06$ \\
\hline $16: 4 n-1$ & $0.34 \pm 0.07$ & $0.55 \pm 0.15$ & $0.73 \pm 0.03$ & $0.66 \pm 0.03$ & $0.39 \pm 0.12$ & $0.32 \pm 0.05$ & $0.62 \pm 0.07$ \\
\hline $18: 2 n-6$ & $1.09 \pm 0.01$ & $1.36 \pm 0.34$ & $1.10 \pm 0.10$ & $1.10 \pm 0.04$ & $1.23 \pm 0.33$ & $1.01 \pm 0.10$ & $1.14 \pm 0.30$ \\
\hline $18: 3 n-3$ & $0.46 \pm 0.06$ & $0.49 \pm 0.10$ & $0.48 \pm 0.02$ & $0.48 \pm 0.01$ & $0.51 \pm 0.08$ & $0.53 \pm 0.03$ & $0.48 \pm 0.07$ \\
\hline $18: 4 n-3$ & $0.65 \pm 0.12$ & $0.97 \pm 0.21$ & $1.13 \pm 0.03$ & $1.13 \pm 0.02$ & $0.89 \pm 0.14$ & $0.87 \pm 0.06$ & $1.04 \pm 0.07$ \\
\hline $20: 2 n-6$ & $0.13 \pm 0.01$ & $0.23 \pm 0.05$ & $0.17 \pm 0.02$ & $0.18 \pm 0.01$ & $0.26 \pm 0.05$ & $0.26 \pm 0.03$ & $0.20 \pm 0.03$ \\
\hline $20: 4 n-3$ & $0.32 \pm 0.04$ & $0.40 \pm 0.08$ & $0.39 \pm 0.02$ & $0.39 \pm 0.02$ & $0.40 \pm 0.07$ & $0.37 \pm 0.03$ & $0.44 \pm 0.07$ \\
\hline $20: 4 n-6$ & $0.57 \pm 0.03$ & $0.55 \pm 0.04$ & $0.50 \pm 0.03$ & $0.61 \pm 0.01$ & $0.74 \pm 0.09$ & $0.96 \pm 0.04$ & $0.67 \pm 0.03$ \\
\hline $20: 5 n-3^{*}$ & $5.73 \pm 0.80$ & $6.23 \pm 1.24$ & $7.54 \pm 0.32$ & $7.74 \pm 0.32$ & $6.66 \pm 0.66$ & $7.50 \pm 0.42$ & $7.83 \pm 0.35$ \\
\hline $21: 5 n-3$ & $0.33 \pm 0.04$ & $0.31 \pm 0.04$ & $0.35 \pm 0.00$ & $0.38 \pm 0.01$ & $0.33 \pm 0.02$ & $0.34 \pm 0.02$ & $0.38 \pm 0.01$ \\
\hline $22: 5 n-3$ & $3.09 \pm 0.20$ & $1.80 \pm 0.21$ & $2.15 \pm 0.09$ & $2.38 \pm 0.09$ & $2.55 \pm 0.40$ & $3.24 \pm 0.35$ & $2.41 \pm 0.14$ \\
\hline $22: 6 n-3^{*}$ & $6.41 \pm 0.36$ & $5.48 \pm 0.63$ & $6.02 \pm 0.08$ & $6.83 \pm 0.09$ & $8.33 \pm 1.29$ & $10.98 \pm .44$ & $7.44 \pm 0.46$ \\
\hline Total SAT & $13.77 \pm 0.76$ & $20.64 \pm 1.24$ & $20.57 \pm 1.01$ & $19.17 \pm 0.44$ & $19.28 \pm 1.28$ & $18.73 \pm 0.51$ & $19.05 \pm 0.93$ \\
\hline Total MUFA & $64.77 \pm 1.56$ & $58.28 \pm 3.71$ & $56.01 \pm 1.23$ & $56.15 \pm 2.82$ & $55.74 \pm 2.25$ & $52.07 \pm 0.87$ & $55.45 \pm 0.90$ \\
\hline Total PUFA & $21.21 \pm 1.40$ & $20.75 \pm 2.79$ & $23.14 \pm 0.34$ & $24.41 \pm 0.31$ & $24.68 \pm 1.80$ & $28.94 \pm 0.78$ & $25.21 \pm 1.03$ \\
\hline
\end{tabular}

guishable from baseline values at Day 0 (Biopsy A) and one sample that was classified together with the herring (Biopsy C) group (Fig. 3).

\section{CC and FA subset model optimization}

The CC values developed from the herring fed juvenile harbour seals ranged from 0.23 for $24: 1 \mathrm{n}-9$ to 12.21 for 14:1n-5 (Table 4). A ratio of 1:1 indicated no FA modification occurred during deposition as the proportion of a particular FA was equal in both the herring and the blubber. Approximately $40 \%$ (27 of 68 FAs) were within $0.9: 1$ to $1.1: 1$ while $66 \%$ (45 of 68 FAs) were within $0.5: 1$ to $1.5: 1$. Our harbour seal CCs generally followed the patterns of published juvenile phocid CCs more so than the phocid pup CCs determined by Iverson et al. (2004) (Table 4). Many of the pup CCs were closer to parity, indicating limited FA modification or biosynthesis following ingestion, while the juvenile phocid CCs had greater variability. Notably, some harbour seal CC values differed by greater than 2-fold for some FA used in each modelling subset including 18:1n-9 and 20:1n-11 (lower than grey and 


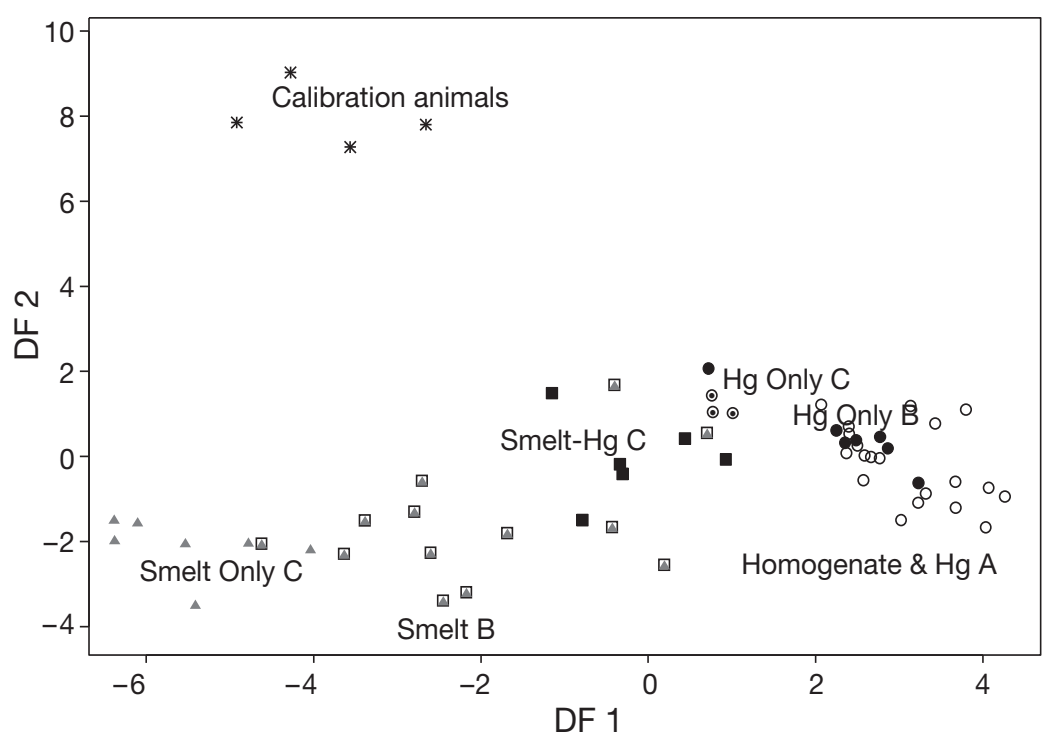

Fig. 3. Phoca vitulina. DFA plot of seal blubber samples based on 6 FA (16:0, $16: 1 \mathrm{n}-7,18: 1 \mathrm{n}-9,20: 1 \mathrm{n}-9,20: 5 \mathrm{n}-3,22: 6 \mathrm{n}-3)$. Letters indicate biopsy period $(\mathrm{A}=$ Day $0, \mathrm{~B}=$ Day 21, C = Day 42) and Hg = herring diet. DFs 1 and 2 accounted for $91.7 \%$ of the total variance. Biopsy groupings were separated with $85 \%$ of original grouped cases and $80 \%$ of cross-validated grouped cases classified correctly harp seal CCs) as well as 22:4n-6 (higher than all published CC sets). Two-fold differences were also observed for $18: 3 n-3,22: 1 n-11,22: 1 n-9$, $22: 1 n-7$ and $22: 4 n-3$ from various individual CC sets (Table 4).

Diet estimates generated using harbour seal calibrations consistently had fewer prey misclassifications than did estimates using grey seal pup CCs (Fig. 4). Misclassifications ranged from $13 \%$ (Reduced) to $21 \%$ (Extended Dietary) when harbour seal CCs were applied to the 59 study biopsies. Misclassifications from estimates using grey seal pup CCs were nearly double in comparison and ranged from $24 \%$ (Reduced) to $42 \%$ (Extended Dietary). Mean misclassification levels for each CC-subset comparison had wide confidence limits (Fig. 4), mainly due to misclassification variability at certain biopsy periods or treatment groups (see next).

Table 4. Phoca vitulina, Halichoerus grypus and P. groenlandica. Calibration coefficients (CC) estimated for 68 fatty acids (FA) and 3 modelling subsets (Reduced, Dietary, Extended Dietary) tested in the study. Phocid derived CCs are from fish-fed juvenile harbour seals, present study (Harbour), grey seals (Grey) and harp seals (Harp), as well as suckling grey seal pups (Grey pup). *Values from Iverson et al. (2004)

\begin{tabular}{|c|c|c|c|c|c|c|c|}
\hline Fatty acid & $\begin{array}{c}\text { Harbour } \\
\text { CC } \\
\mathrm{N}=4\end{array}$ & $\begin{array}{l}\text { Grey } \\
\mathrm{CC}^{*} \\
\mathrm{~N}=8\end{array}$ & $\begin{array}{l}\text { Harp } \\
\mathrm{CC}^{*} \\
\mathrm{~N}=5\end{array}$ & $\begin{array}{l}\text { Grey pup } \\
\mathrm{CC}^{*} \\
\mathrm{~N}=17\end{array}$ & $\begin{array}{c}\text { Reduced } \\
\text { FA subset } \\
\mathrm{N}=35 \text { FAs }\end{array}$ & $\begin{array}{c}\text { Dietary } \\
\text { FA subset } \\
\mathrm{N}=33 \text { FAs }\end{array}$ & $\begin{array}{c}\text { Extended Dietary } \\
\text { FA Subset } \\
\text { N = 41 FAs }\end{array}$ \\
\hline $12: 0$ & 1.32 & 0.97 & 0.86 & 0.92 & & & \\
\hline 13.0 & 1.00 & 1.00 & 1.00 & 1.00 & & & \\
\hline iso-14:0 & 1.27 & 1.00 & 1.00 & 1.00 & & & \\
\hline $14: 0$ & 0.91 & 0.86 & 0.93 & 0.95 & & & $\mathrm{X}$ \\
\hline $14: 1 n-9$ & 0.84 & 0.70 & 1.06 & 0.75 & & & \\
\hline $14: 1 \mathrm{n}-7$ & 2.53 & 1.14 & 1.03 & 1.26 & & & \\
\hline $14: 1 \mathrm{n}-5$ & 12.21 & 10.92 & 8.83 & 1.54 & & & \\
\hline iso-15:0 & 1.02 & 1.12 & 1.11 & 0.91 & & & \\
\hline anteiso-15:0 & 1.41 & 1.30 & 0.95 & 0.84 & & & \\
\hline $15: 0$ & 0.96 & 1.09 & 0.97 & 0.97 & & & \\
\hline $15: 1 n-8$ & 0.95 & 1.00 & 1.00 & 1.00 & & & \\
\hline $15: 1 n-6$ & 5.53 & 1.24 & 1.00 & 1.20 & & & \\
\hline iso-16:0 & 0.90 & 1.16 & 0.82 & 0.96 & & & \\
\hline $16: 0$ & 0.64 & 0.74 & 0.63 & 0.83 & & & $\mathrm{X}$ \\
\hline 16:1n-11 & 1.65 & 2.51 & 2.24 & 0.98 & & & \\
\hline $16: 1 n-9$ & 2.28 & 3.37 & 2.64 & 1.11 & & & \\
\hline $16: 1 n-7$ & 2.34 & 1.52 & 1.61 & 1.30 & & & $\mathrm{X}$ \\
\hline 7methyl16:0 & 1.11 & 1.10 & 1.08 & 1.04 & & & \\
\hline $16: 1 n-5$ & 1.60 & 1.12 & 1.05 & 1.01 & & & \\
\hline $16: 2 n-6$ & 0.89 & 0.76 & 0.74 & 0.81 & $\mathrm{X}$ & $\mathrm{X}$ & $\mathrm{X}$ \\
\hline iso- $17: 0$ & 0.96 & 1.09 & 1.05 & 0.96 & & & \\
\hline $16: 2 n-4$ & 1.13 & 1.50 & 0.95 & 0.89 & $\mathrm{X}$ & $\mathrm{X}$ & $\mathrm{X}$ \\
\hline $16: 3 n-6$ & 0.85 & 0.86 & 1.12 & 1.00 & $\mathrm{X}$ & $\mathrm{X}$ & $\mathrm{X}$ \\
\hline $17: 0$ & 0.42 & 1.40 & 0.91 & 0.78 & & & $X$ \\
\hline $16: 3 n-4$ & 0.75 & 0.68 & 0.87 & 0.98 & $\mathrm{X}$ & $\mathrm{X}$ & $\mathrm{X}$ \\
\hline $17: 1$ & 1.40 & 2.67 & 2.04 & 1.27 & & & \\
\hline $16: 3 n-1$ & 1.00 & 0.85 & 0.57 & 1.14 & $\mathrm{X}$ & $\mathrm{X}$ & $\mathrm{X}$ \\
\hline $16: 4 n-3$ & 0.99 & & & 0.90 & $\mathrm{X}$ & $\mathrm{X}$ & $\mathrm{X}$ \\
\hline
\end{tabular}


Table 4 (continued)

\begin{tabular}{|c|c|c|c|c|c|c|c|}
\hline Fatty acid & $\begin{array}{l}\text { Harbour } \\
\text { CC } \\
\mathrm{N}=4\end{array}$ & $\begin{array}{l}\text { Grey } \\
\mathrm{CC}^{*} \\
\mathrm{~N}=8\end{array}$ & $\begin{array}{l}\text { Harp } \\
\mathrm{CC}^{*} \\
\mathrm{~N}=5\end{array}$ & $\begin{array}{c}\text { Grey pup } \\
\text { CC }^{*} \\
\mathrm{~N}=17\end{array}$ & $\begin{array}{c}\text { Reduced } \\
\text { FA subset } \\
\mathrm{N}=35 \text { FAs }\end{array}$ & $\begin{array}{c}\text { Dietary } \\
\text { FA subset* } \\
\mathrm{N}=33 \text { FAs }\end{array}$ & $\begin{array}{c}\text { Extended Dietary } \\
\text { FA Subset } \\
\text { N }=41 \text { FAs }\end{array}$ \\
\hline $16: 4 n-1$ & 0.74 & 0.59 & 0.77 & 0.97 & $\mathrm{X}$ & $\mathrm{X}$ & $\mathrm{X}$ \\
\hline $18: 0$ & 0.54 & 0.84 & 0.79 & 0.64 & & & $\mathrm{X}$ \\
\hline 18:1n-13 & 2.95 & 0.95 & 0.74 & 0.89 & & & \\
\hline 18:1n-11 & 6.35 & 15.04 & 10.40 & 1.04 & & & \\
\hline 18:1n-9 & 1.25 & 3.46 & 2.79 & 1.15 & $\mathrm{X}$ & & $\mathrm{X}$ \\
\hline $18: 1 n-7$ & 1.10 & 1.41 & 1.44 & 1.04 & $\mathrm{X}$ & & $\mathrm{X}$ \\
\hline $18: 1 n-5$ & 1.05 & 1.04 & 1.00 & 0.99 & & & \\
\hline $18: 2-5,11$ & 1.46 & 1.04 & 1.00 & 0.87 & & & \\
\hline $18: 2 n-7$ & 2.04 & 1.13 & 1.00 & 1.26 & & & \\
\hline $18: 2 n-6$ & 1.42 & 2.02 & 1.57 & 1.04 & $\mathrm{X}$ & $\mathrm{X}$ & $\mathrm{X}$ \\
\hline $18: 2 n-4$ & 1.09 & 0.98 & 0.86 & 0.94 & $\mathrm{X}$ & $\mathrm{X}$ & $\mathrm{X}$ \\
\hline $18: 3 n-6$ & 0.90 & 1.08 & 0.94 & 0.78 & $\mathrm{X}$ & $\mathrm{X}$ & $\mathrm{X}$ \\
\hline $18: 3 n-4$ & 1.92 & 2.32 & 2.59 & 1.01 & $\mathrm{X}$ & $\mathrm{X}$ & $\mathrm{X}$ \\
\hline $18: 3 n-3$ & 1.30 & 2.27 & 1.48 & 1.07 & $\mathrm{X}$ & $\mathrm{X}$ & $\mathrm{X}$ \\
\hline $18: 3 n-1$ & 1.10 & 0.95 & 0.95 & 0.88 & $\mathrm{X}$ & $\mathrm{X}$ & $\mathrm{X}$ \\
\hline $18: 4 n-3$ & 0.90 & 0.96 & 0.99 & 0.96 & $\mathrm{X}$ & $\mathrm{X}$ & $\mathrm{X}$ \\
\hline $18: 4 n-1$ & 1.77 & 1.10 & 1.39 & 1.01 & $\mathrm{X}$ & $\mathrm{X}$ & $\mathrm{X}$ \\
\hline $20: 0$ & 0.55 & 0.50 & 0.50 & 1.00 & & & \\
\hline $20: 1 n-11$ & 1.80 & 3.42 & 2.83 & 0.97 & $\mathrm{X}$ & $\mathrm{X}$ & $\mathrm{X}$ \\
\hline $20: 1 n-9$ & 0.86 & 0.81 & 1.00 & 0.91 & $\mathrm{X}$ & $\mathrm{X}$ & $\mathrm{X}$ \\
\hline $20: 1 n-7$ & 1.05 & 0.71 & 1.05 & 0.82 & $\mathrm{X}$ & $\mathrm{X}$ & $\mathrm{X}$ \\
\hline $20: 2 n-9$ & 0.83 & 1.00 & 2.93 & 1.00 & & & \\
\hline $20: 2 n-6$ & 1.33 & 1.65 & 1.39 & 1.02 & $\mathrm{X}$ & $\mathrm{X}$ & $\mathrm{X}$ \\
\hline $20: 3 n-6$ & 1.00 & 1.07 & 1.00 & 0.91 & $\mathrm{X}$ & $\mathrm{X}$ & $\mathrm{X}$ \\
\hline $20: 4 n-6$ & 1.14 & 0.82 & 1.04 & 0.92 & $\mathrm{X}$ & $\mathrm{X}$ & $\mathrm{X}$ \\
\hline $20: 3 n-3$ & 1.25 & 1.16 & 0.98 & 0.98 & $\mathrm{X}$ & $\mathrm{X}$ & $\mathrm{X}$ \\
\hline $20: 4 n-3$ & 1.36 & 2.11 & 1.50 & 1.00 & $\mathrm{X}$ & $\mathrm{X}$ & $\mathrm{X}$ \\
\hline $20: 5 n-3$ & 0.75 & 0.65 & 0.80 & 0.82 & $\mathrm{X}$ & $\mathrm{X}$ & $\mathrm{X}$ \\
\hline $22: 1 n-11$ & 0.42 & 0.20 & 0.34 & 0.47 & $\mathrm{X}$ & $\mathrm{X}$ & $\mathrm{X}$ \\
\hline $22: 1 n-9$ & 0.57 & 0.27 & 0.59 & 0.49 & $\mathrm{X}$ & $\mathrm{X}$ & $\mathrm{X}$ \\
\hline $22: 1 n-7$ & 0.33 & 0.18 & 0.26 & 0.90 & $\mathrm{X}$ & $\mathrm{X}$ & $\mathrm{X}$ \\
\hline $22: 2 n-6$ & 1.04 & 1.00 & 1.00 & 1.00 & $\mathrm{X}$ & $\mathrm{X}$ & $\mathrm{X}$ \\
\hline $21: 5 n-3$ & 1.02 & 1.37 & 1.45 & 1.02 & $\mathrm{X}$ & $\mathrm{X}$ & $\mathrm{X}$ \\
\hline $22: 4 n-6$ & 2.43 & 1.00 & 1.00 & 1.03 & $\mathrm{X}$ & $\mathrm{X}$ & $\mathrm{X}$ \\
\hline $22: 5 n-6$ & 1.23 & 1.04 & 0.76 & 0.96 & $\mathrm{X}$ & $\mathrm{X}$ & $\mathrm{X}$ \\
\hline $22: 4 n-3$ & 1.51 & 2.58 & 1.55 & 1.01 & $\mathrm{X}$ & $\mathrm{X}$ & $\mathrm{X}$ \\
\hline $22: 5 n-3$ & 2.98 & 4.64 & 3.91 & 1.09 & & & $\mathrm{X}$ \\
\hline $22: 6 n-3$ & 1.01 & 1.11 & 0.93 & 1.00 & $\mathrm{X}$ & $\mathrm{X}$ & $\mathrm{X}$ \\
\hline $24: 1 n-9$ & 0.23 & 0.13 & 0.15 & 0.32 & & & \\
\hline
\end{tabular}

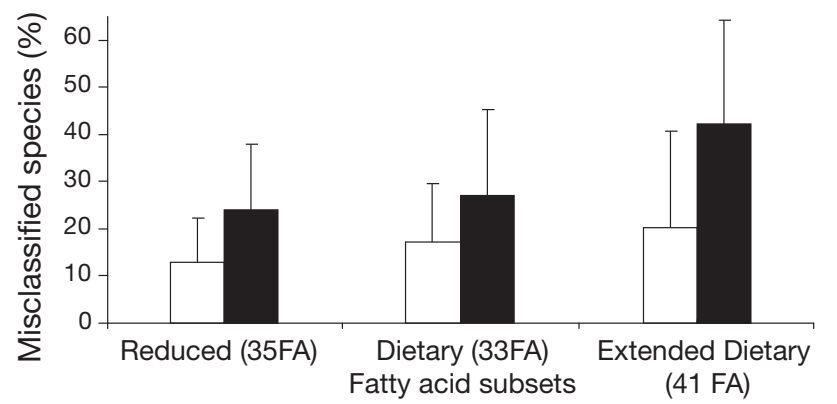

Fig. 4. Phoca vitulina and Halichoerus grypus. Percentage of misclassified prey (mean $+95 \%$ CI) in QFASA diet estimates from feeding study biopsies $(\mathrm{N}=57)$ when using different calibration coefficients for 3 FA subsets (see Table 4 for subset descriptions). Bars indicate calibration set used (white $=$ juvenile harbour seal, black = grey seal pup); 11-species prey library was used in the model
The Reduced FA subset (Table 4) was the newly developed subset that generated the fewest misclassifications overall during preliminary optimization and was subsequently compared with the Dietary and the Extended Dietary subsets (Fig. 5). There were no significant differences between the FA subsets at Biopsy A (ANOVA, $\left.F_{2,18}<1.93, \mathrm{p}>0.175\right)$ in the low level of other species falsely reported (Fig. 5). However, the choice of FA subset significantly affected the proportion of misclassified (false positive) prey identified at both Biopsy B and Biopsy C (ANOVA, F > 7.20, p < 0.003 for all comparisons). Post hoc comparisons revealed the Dietary and the Extended Dietary subsets produced significantly more misclassified prey than the Reduced subset although this was dependent on the biopsy periods and dietary treatments compared 


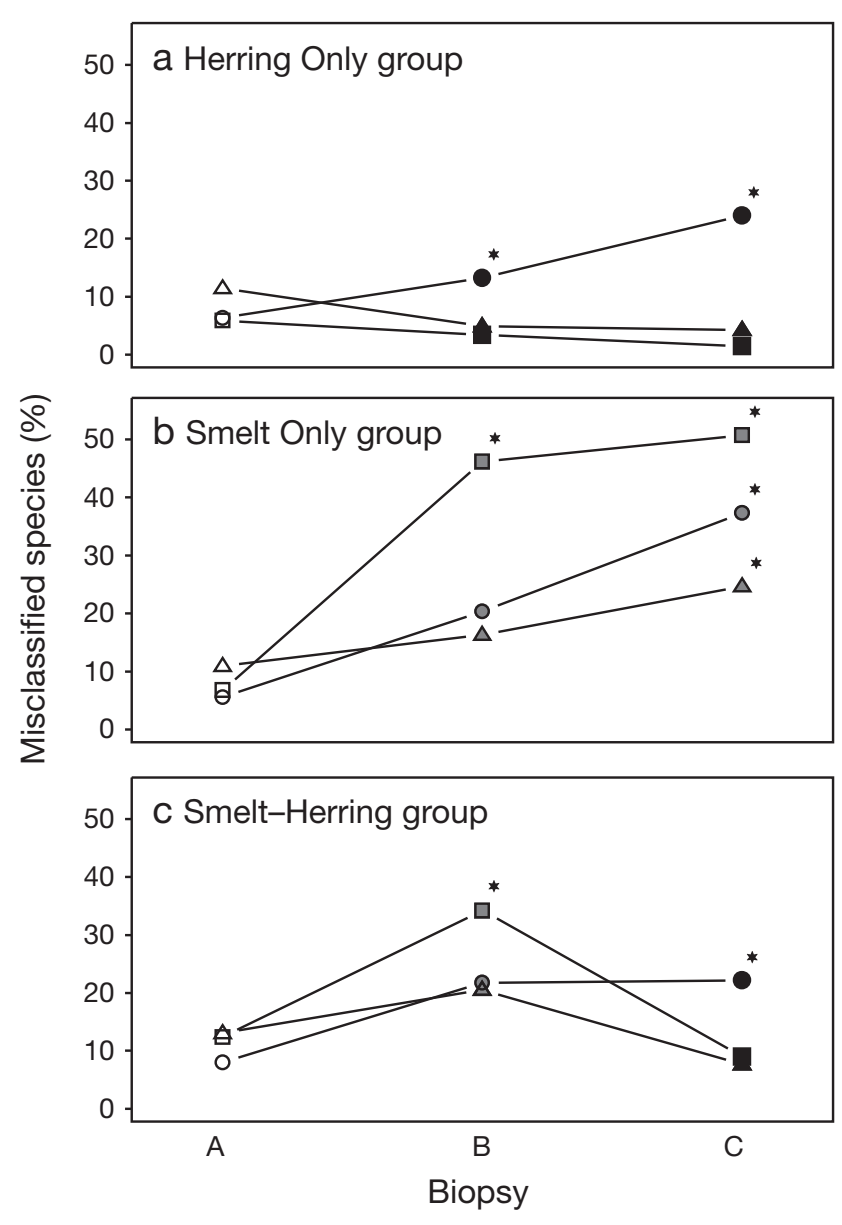

Fig. 5. Phoca vitulina. Mean percent of relative misclassified prey in QFASA diet estimates using the Reduced (triangles), Dietary (circles) and Extended Dietary (squares) FA subsets (see Table 4) for the (a) Herring Only, (b) Smelt Only, and (c) Smelt-Herring treatment groups. *Significant differences between FA subsets at each biopsy period (Holm-Sidak method). Symbol shading indicates previous diet (white $=$ homogenate and herring, black $=$ herring, grey $=$ smelt). Harbour seal CC and 11-species prey library were used in the model

(Holm-Sidak method, $t<15.21, \mathrm{p}<0.01$; Fig. 5). Overall, mean false positive rates ranged from $1 \%$ in the Herring Only group (Biopsy C, Extended Dietary subset) to as high as $51 \%$ in the Smelt Only group (Biopsy C, Extended Dietary subset) when using the 11 prey species library.

\section{QFASA model evaluation post optimization}

The QFASA diet estimates were generated using optimized parameters (i.e. harbour seal CCs and the Reduced FA subset). Model diet estimates for each diet group varied with the number of species included in the potential prey library (Fig. 6). When using the full 11-species prey library, QFASA estimated a diet of primarily herring for all 21 seals at the start of the study (Biopsy A; Fig. 6). Subsequent estimates at Biopsy B and Biopsy $\mathrm{C}$ were generally consistent with tracking changes in experimental feeding regimes (Fig. 6). For example, QFASA correctly estimated consistently high amounts of herring (>95\%) for the Herring Only group, consecutive increases in amount of smelt in the Smelt Only group (from $55 \%$ to $60 \%$ ), and an increase $(39 \%)$ followed by a decrease $(13 \%)$ in the proportion of smelt in the Smelt-Herring group (Fig. 6). Misclassified species had an overall mean of $12 \%$ (range, 4 to $25 \%$ ).

Reducing the number of potential prey species in the model library from 11 to 10 to 3 species decreased the proportion of misclassified other prey (Fig. 6), but otherwise overall diet estimates were similar among the 3 prey library sets. Using 10 species in the prey library (coho salmon removed), the model estimated an increased proportion of smelt at Biopsy B (3 to 11\%) and Biopsy C (1 to $13 \%$ ) for the seals that consumed smelt, but it had no effect on herring estimates for any group. Restricting the model to the 3-species prey library (i.e. just those species fed) resulted in minor increases in the amount of herring estimated for all groups (1 to $5 \%$ ), but greater increases in the amount of smelt estimated for the smelt eating treatment groups at Biopsy B (15 to 22\%) and Biopsy C (3 to $24 \%)$.

Misclassification rates for each biopsy period and diet treatment were specific to the prey library employed and, as expected, were highest when using the largest prey library. Mean estimates ranged from $4 \%$ (Herring Only, Biopsy C, Fig. 6a) to $25 \%$ misclassified species (Smelt Only, Biopsy C, Fig. 6c). Specific prey misclassifications included capelin selected instead of herring (mean error $=10 \%$, range $=3$ to $18 \%, N=6$ biopsies), as well as coho salmon (mean error $=15 \%$, range $=1$ to $28 \%, N=15$ biopsies) and to a lesser degree sandlance (mean error $=5 \%$, range $=1$ to $8 \%, N=16$ biopsies) selected in lieu of smelt. Misclassification rates for diet groups consuming smelt were significantly higher using published FA subsets.

Comparing herring and smelt QFASA diet estimates across biopsies (i.e. from A, to B, to C) within treatments showed overall significant statistical differences for the Smelt Only and Smelt-Herring groups (Table 5). Smelt Only biopsies were significantly different in the amount of herring $(\mathrm{p}<0.001)$ and smelt $(p<0.001)$ estimated by the model over time as were the Smelt-Herring biopsies $(\mathrm{p}<0.005$ and $\mathrm{p}<0.01$, respectively). Biopsies from the Herring Only group were not significantly different over the course of the experiment $(p=0.07)$, which was consistent with the seals being maintained on an unchanging diet. 
a Herring Only Biopsy A

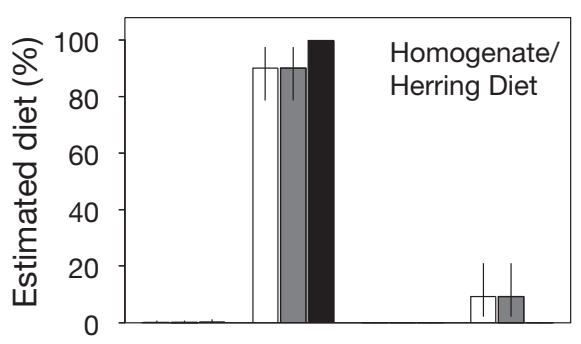

b Smelt Only

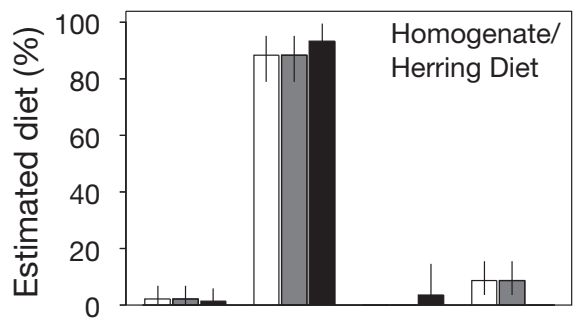

C Smelt-Herring

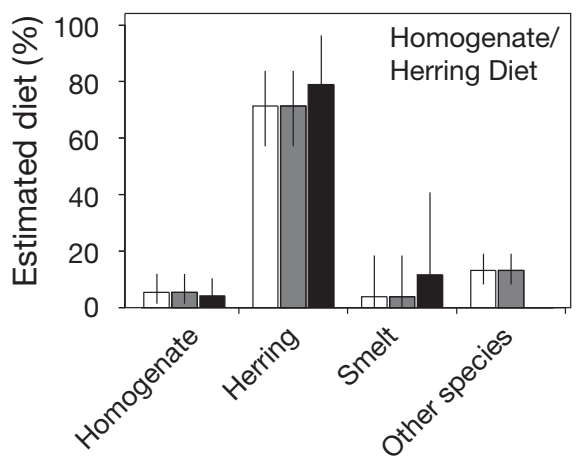

Biopsy B
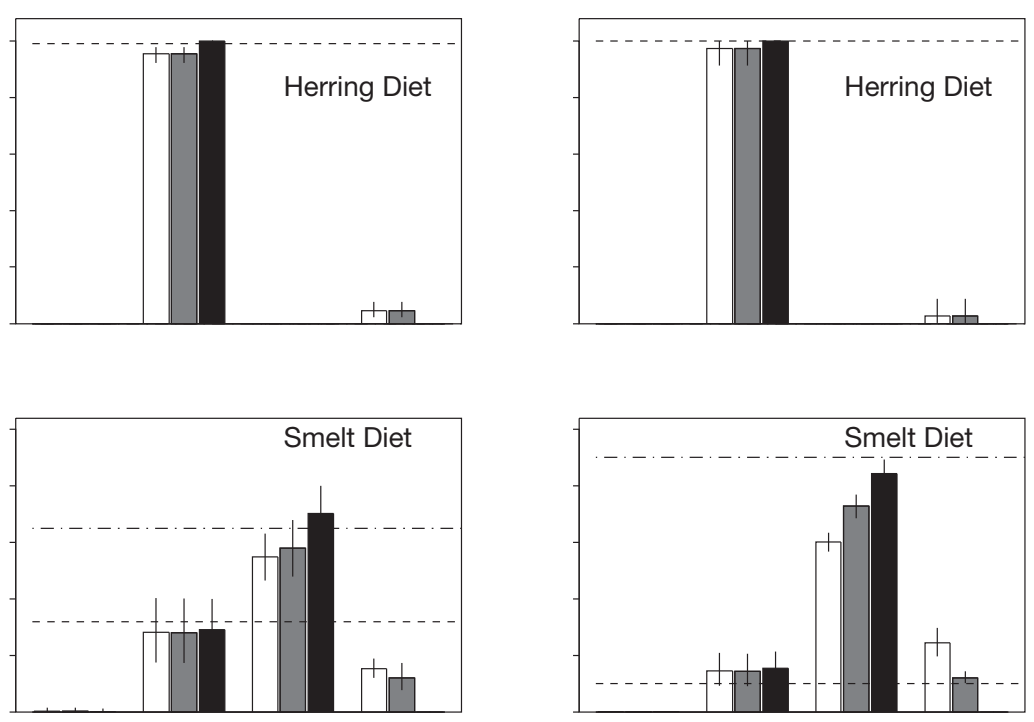

Biopsy C

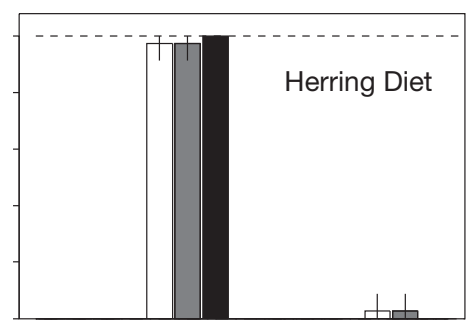

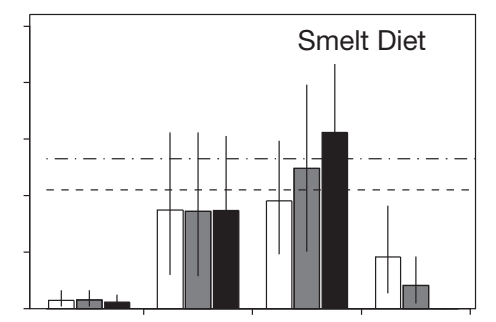

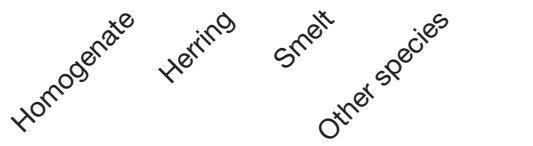

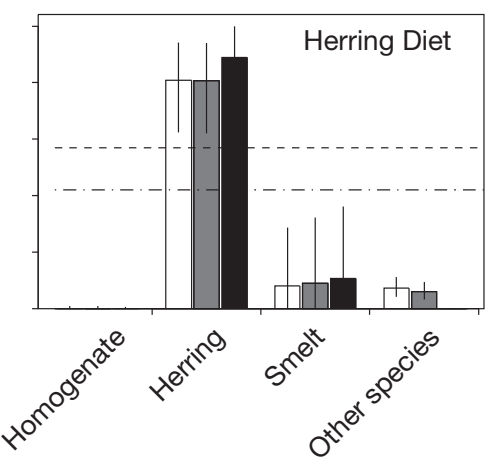

Diet components

Fig. 6. Phoca vitulina. Percent of QFASA diet estimates (mean \pm CI) for harbour seals in the (a) Herring Only, (b) Smelt Only and (c) Smelt-Herring treatment groups using harbour seal CC and the Reduced FA subset (35 FAs). The 3 bars for each diet component represent prey libraries used with the model: white = all available (11 species), grey = coho salmon removed (10 species), black $=$ known fed prey ( 3 species). See Table 5 for statistical comparisons. Horizontal lines represent expected herring (- - ) and smelt (-.-) proportions from recorded diet accumulated $55 \mathrm{~d}$ prior to each biopsy

Comparing herring and smelt QFASA diet estimates across diet treatments (i.e. between Herring Only and Smelt Only groups) showed significant differences within biopsy periods overall (Table 5). Herring estimates were significantly different $(p<0.02)$ at the onset of the experiment (Biopsy A), consistent with the variable, weaning time, for the seals. Smelt estimates did not differ significantly across the diet groups $(\mathrm{p}=$ 0.05) at Biopsy A despite a limited amount of smelt being estimated in the diet before it was fed to the seals (Fig. 6). Herring and smelt estimates at Biopsy B were significantly different overall $(\mathrm{p}<0.001)$ with post hoc comparisons noting that the 2 smelt fed groups ( $21 \mathrm{~d}$ ) were significantly different from the Herring Only group, but not from each other. Diet esti- mates of both herring and smelt (Fig. 6) were significantly different for all treatment groups at Biopsy C $(\mathrm{p}<0.001)$.

Variance analysis showed individual animal QFASA estimates more accurately mirrored fed diet at Biopsy $\mathrm{C}$ after seals had been fed a steady diet (1 species) over the $42 \mathrm{~d}$ study (Fig. 7). The Herring Only group showed no deviation as they had consumed herring exclusively $(100 \%)$ for $>65 \mathrm{~d}$ before the biopsy and QFASA estimated $100 \%$ herring for each seal. Lengthening the recorded feeding period for comparison from 40 to $65 \mathrm{~d}$ also improved deviance from expected and estimated diet (best fit $=65 \mathrm{~d}$ ). Accordingly, diet estimates deviated less for individuals from the Smelt Only group (range $=1$ to $13 \%$ ) than seals from the Smelt- 
Table 5. Phoca vitulina. Mean herring and smelt QFASA diet estimates compared across biospies and diets, using Linear Mixed Effect ANOVA. Three variations of the prey library and post hoc multiple comparisons are included for each comparison (A, B, C = Biopsy A, B and C, respectively; diet treatments: $\mathrm{HG}=$ Herring Only, SM = Smelt Only, S-H = Smelt-Herring; Yes: significant comparison)

\begin{tabular}{|c|c|c|c|c|c|c|c|c|c|}
\hline \multirow{3}{*}{$\begin{array}{l}\text { Group } \\
\text { Biopsy }\end{array}$} & \multirow[t]{3}{*}{ Prey library } & \multicolumn{4}{|c|}{ — Mean herring QFASA diet estimates — } & \multicolumn{4}{|c|}{ - Mean smelt QFASA diet estimates } \\
\hline & & \multirow[t]{2}{*}{$\begin{array}{c}\text { ANOVA values } \\
F(\mathrm{p})\end{array}$} & \multicolumn{3}{|c|}{$\begin{array}{l}\text { Significant Tukey's } \\
\text { multiple comparisons } \\
\text { (Bonferroni corrected) }\end{array}$} & \multirow[t]{2}{*}{$\begin{array}{c}\text { ANOVA values } \\
F(\mathrm{p})\end{array}$} & \multicolumn{3}{|c|}{$\begin{array}{l}\text { Significant Tukey's } \\
\text { multiple comparisons } \\
\text { (Bonferroni corrected) }\end{array}$} \\
\hline & & & A vs. B & A vs. $\mathrm{C}$ & B vs. C & & A vs. B & A vs. $\mathrm{C}$ & B vs. C \\
\hline \multirow[t]{3}{*}{$\mathrm{HG}$} & 11 species & $3.8(0.07)$ & & None & & - & - & - & - \\
\hline & 10 species & $3.8(0.07)$ & & None & & - & - & - & - \\
\hline & 3 species & $1.6(0.25)$ & & None & & - & - & - & - \\
\hline \multirow[t]{3}{*}{$\mathrm{SO}$} & 11 species & $419.8(<0.001)$ & Yes & Yes & Yes & $619.1(<0.001)$ & Yes & Yes & No \\
\hline & 10 species & $423.3(<0.001)$ & Yes & Yes & Yes & $617.5(<0.001)$ & Yes & Yes & Yes \\
\hline & 3 species & $199.1(<0.001)$ & Yes & Yes & Yes & $229.4(<0.001)$ & Yes & Yes & Yes \\
\hline \multirow[t]{3}{*}{$\mathrm{S}-\mathrm{H}$} & 11 species & $12.3(0.001)$ & Yes & Yes & Yes & $6.1(0.01)$ & Yes & No & Yes \\
\hline & 10 species & $12.3(0.001)$ & Yes & Yes & Yes & $8.1(0.01)$ & Yes & No & Yes \\
\hline & 3 species & $8.5(0.005)$ & Yes & Yes & Yes & $6.8(0.01)$ & Yes & No & Yes \\
\hline \multirow{4}{*}{$\begin{array}{l}\text { Diet } \\
\text { A, Day } 0\end{array}$} & & & HG vs. SM & HG vs. S-H & SM vs. S-H & & HG vs. SM & HG vs. S-H & SM vs. S-H \\
\hline & 11 species & $5.9(0.02)$ & Yes & Yes & Yes & $4.1(0.05)$ & & None & \\
\hline & 10 species & $5.9(0.02)$ & Yes & Yes & Yes & $4.1(0.05)$ & & None & \\
\hline & 3 species & $6.8(0.01)$ & Yes & Yes & No & $4.0(0.05)$ & & None & \\
\hline \multirow[t]{3}{*}{ B, Day 21} & 11 species & $52.7(<0.001)$ & Yes & Yes & No & $36.1(<0.001)$ & Yes & Yes & No \\
\hline & 10 species & $51.3(<0.001)$ & Yes & Yes & No & $24.8(<0.001)$ & Yes & Yes & No \\
\hline & 3 species & $30.2(<0.001)$ & Yes & Yes & No & $29.5(<0.001)$ & Yes & Yes & No \\
\hline \multirow[t]{3}{*}{ C, Day 42} & 11 species & $90.0(<0.001)$ & Yes & Yes & Yes & $36.4(<0.001)$ & Yes & Yes & Yes \\
\hline & 10 species & $90.6(<0.001)$ & Yes & Yes & Yes & $42.5(<0.001)$ & Yes & Yes & Yes \\
\hline & 3 species & $48.2(<0.001)$ & Yes & Yes & Yes & $49.0(<0.001)$ & Yes & Yes & Yes \\
\hline
\end{tabular}

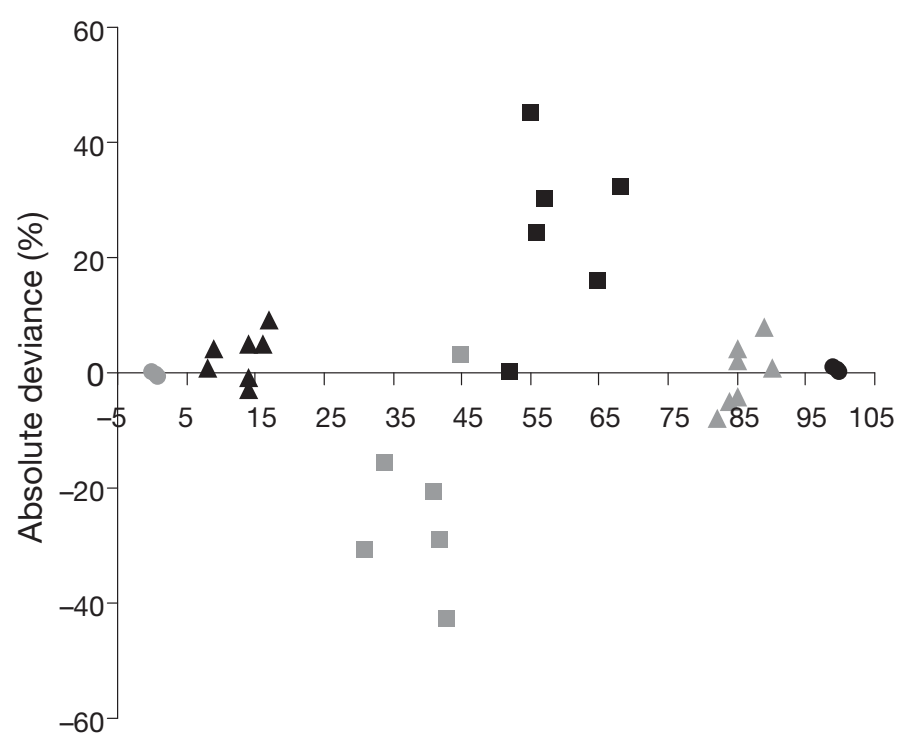

Diet proportion (\%)

Fig. 7. Phoca vitulina. Absolute percent deviation of QFASA diet estimates from recorded diets of harbour seals $65 \mathrm{~d}$ prior to Biopsy $\mathrm{C}$, based on 3 -species prey library. $(\bullet, 0)$ herring and smelt prediction from Herring Only group $(\mathrm{N}=3)$, respectively; $(\boldsymbol{\Lambda}, \mathbf{\Delta})$ herring and smelt prediction from Smelt Only group ( $N=7)$, respectively; $(\boldsymbol{\square}, \mathbf{\square})$ herring and smelt prediction from Smelt-Herring group $(\mathrm{N}=6)$, respectively
Herring group (range $=0$ to $50 \%$ ) as the dietary history became more inclusive of pre-Biopsy A (herring and homogenate) diet. In general, the wet weight proportion of smelt in the diet was underestimated while the proportion of herring was overestimated at Biopsy C for the 13 seals that consumed smelt during the study.

\section{Blubber FA signature turnover}

The first turnover estimate was generated from the percent smelt change detected over the series of biopsies. The QFASA model (3 species library) estimated an increase from 7 to $84 \%$ (77\% total increase) for the Smelt Only group over $42 \mathrm{~d}$. The estimated proportion of smelt increased and then decreased for the Smelt-Herring group while smelt was not detected at any time for the Herring Only group as might be expected over the $42 \mathrm{~d}$ (Fig. 8). Smelt turnover did not occur in a strictly linear fashion as estimates increased by $63 \%$ in the first $21 \mathrm{~d}$ compared with $14 \%$ in the following $21 \mathrm{~d}$ (based on the 3 prey species library) in the Smelt Only group. By directly extrapolating a $77 \%$ increase in $42 \mathrm{~d}$, then estimated time to complete turnover would amount to $\sim 55 \mathrm{~d}$ (Fig. 8). Alternately, if turnover rates do indeed 


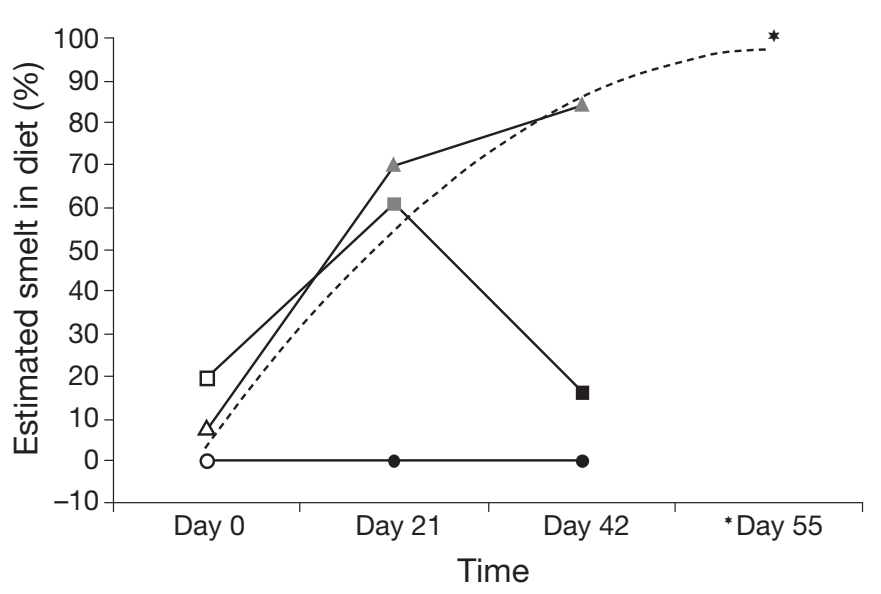

Fig. 8. Phoca vitulina. Mean percentage of smelt in diet estimated for Herring Only (circle symbols), Smelt Only (triangle symbols), and Smelt-Herring (square symbols) treatment groups over $42 \mathrm{~d}$ using the 3 prey species library in the QFASA model. *Minimum projected time for complete turnover in the Smelt Only group (assuming steady turnover) included for comparison. Symbol shading indicates previous diet (white $=$ homogenate and herring, black $=$ herring, grey $=$ smelt)

slow down with time, then turnover may extend well beyond $55 \mathrm{~d}$. The $21 \mathrm{~d}$ turnover of smelt in the Smelt-Herring diet group was somewhat slower in comparison and probably reflected lower smelt intake with an initial $41 \%$ increase between Biopsy $\mathrm{A}$ and Biopsy B followed by a $45 \%$ decrease between Biopsy B and Biopsy C. Alternate turnover estimates

Table 6. Phoca vitulina. Fatty acid turnover estimates for harbour seal blubber in this study based on chi-square analysis. Non-significant results in bold type indicate no difference between QFASA estimated diet (3-species prey library) and recorded diet at 35 to $75 \mathrm{~d}$ (where applicable) prior to Biopsy B and Biopsy C

\begin{tabular}{|c|c|c|c|c|c|c|c|}
\hline \multirow[t]{2}{*}{ Biopsy } & \multirow{2}{*}{$\begin{array}{l}\text { Days } \\
\text { before } \\
\text { biopsy }\end{array}$} & \multicolumn{3}{|c|}{ Smelt Only group } & \multicolumn{3}{|c|}{ Smelt-Herring group } \\
\hline & & $\begin{array}{l}\text { Homo- } \\
\text { genate }\end{array}$ & Herring & Smelt & $\begin{array}{l}\text { Homo- } \\
\text { genate }\end{array}$ & Herring & Smelt \\
\hline \multirow[t]{5}{*}{ B } & 35 & 0.963 & $<0.001$ & 0.017 & 0 & $<0.001$ & $<0.001$ \\
\hline & 40 & 0.650 & $<0.001$ & 0.096 & 0 & $<0.001$ & $<0.001$ \\
\hline & 45 & 0.326 & 0.003 & 0.268 & 0.012 & $<0.001$ & $<0.001$ \\
\hline & 50 & 0.174 & 0.009 & 0.293 & 0.514 & 0.008 & 0.036 \\
\hline & 55 & 0 & 0.017 & 0.373 & 0.552 & 0.020 & 0.079 \\
\hline \multirow[t]{9}{*}{$\mathrm{C}$} & 35 & $<0.001$ & 0 & 0.003 & 0 & 0 & 0 \\
\hline & 40 & $<0.001$ & 0 & 0.003 & 0 & 0 & 0 \\
\hline & 45 & $<0.001$ & 0 & 0.040 & 0 & 0 & 0 \\
\hline & 50 & 0.996 & $<0.001$ & 0.299 & 0 & $<0.001$ & 0 \\
\hline & 55 & 0.980 & $<0.001$ & 0.686 & 0 & $<0.001$ & 0 \\
\hline & 60 & 0.783 & 0.017 & 0.897 & 0 & $<0.001$ & $<0.001$ \\
\hline & 65 & 0.402 & 0.100 & 0.955 & 0 & $<0.001$ & $<0.001$ \\
\hline & 70 & 0.560 & 0.444 & 0.981 & 0 & $<0.001$ & $<0.001$ \\
\hline & 75 & 0.601 & 0.565 & 0.990 & 0 & $<0.001$ & $<0.001$ \\
\hline
\end{tabular}

were based on agreement between QFASA diet estimates and cumulative recorded intake, such that a match (non-significant chi-square tests) at a given time period assumed that an appropriate diet history period had been selected. Non-significant differences were observed at various time intervals for homogenate, herring and smelt (Table 6). Overall, overlap across all prey, treatment groups and biopsy periods appeared strongest at 50 to $65 \mathrm{~d}$. There were no matches in the Smelt-Herring group at Biopsy $\mathrm{C}$ as the difference between expected and observed diets was too great (Table 6; Fig. 6).

\section{DISCUSSION}

Our 42 d captive feeding study demonstrated that QFASA can correctly distinguish seals on single species substitution diets. Reliability of the model was strongly influenced by the CC set, the FA subset and, to a lesser extent, by the species composition of the prey library. Optimal diet estimates were obtained using the harbour seal CC, the newly developed Reduced FA subset (Table 4) and the 3 known prey fed as parameters in the model. Prey misclassifications varied across diet regime, with lowest levels for the pure herring diet, but averaged only $12 \%$ (range $=4$ to $25 \%$ ) overall when all unfed prey were included in the library. The QFASA estimates and fed diet appeared in closest agreement when diet from $\sim 1.5$ to 3 mo prior were integrated. Overall, the harbour seal CCs generated in this study were similar to those previously reported for juvenile phocids (Iverson et al. 2004); however, 2 -fold differences were observed in some FA (Table 4). It is uncertain whether these differences were due to species effects or to our considerably longer monotypic feeding period, and thus our potentially more accurate, herring-diet derived CC. Given that this captive study represented optimal conditions, we believe the results provide important insights and define realistic limits for researchers aiming to study the diet composition of freeranging seals from blubber samples.

\section{Qualitative separation of prey and blubber samples}

Prey classification success was generally robust using DFA techniques with most prey clustering separately 
using only the DFs 1 and 2. The small size class of smelt was an exception as smelt clustered with coho salmon and to a minor extent with sandlance (Fig. 2). The overlap probably explains the relatively high number of misclassifications of smelt with those prey species in the QFASA model. Techniques similar to DFA are clearly an important precursor to using the QFASA model to determine which potential prey species may have analogous FA signatures and have particular relevance here as the overlapping species are known prey of the Pacific harbour seal (Olesiuk et al. 1990, Browne et al. 2002). Our blubber DFA analyses highlighted that animal diet groupings were discernable with 80 to $85 \%$ accuracy using just 6 FAs, particularly between the 4 calibration seals that were fed herring for $>1 \mathrm{yr}$ and the 7 seals that consumed smelt for the entire study (Fig. 3). Minor misclassifications between remaining groupings were generally understandable and were consistent to groupings with high diet overlap (e.g. Herring Only Biopsy B misclassified as Herring Only Biopsy C).

\section{CC and FA subset modeling optimization}

Misclassification levels using our calibrations based on juvenile harbour seals were approximately half that of those when published grey seal pup CCs were substituted (Fig. 4) and results were consistent irrespective of the FA subset employed. While the newly weaned harbour seals undertaking this study were similar in age to the grey seal pups ( 2 wk old), we suspect their FA dynamics were more comparable with the juvenile harbour seals as they were both on a fish diet. Grey pup CCs were based on an exceedingly high (i.e. $60 \%$ ) fat milk, which probably suppressed FA biosynthesis completely (Iverson et al. 2004) and may explain why most grey pup CCs were close to 1.0 (Table 4). In contrast, seals consuming fish, even at a young age, appear to biosynthesize and metabolize various FA in addition to those from dietary sources. Accordingly, their blubber was better modelled using the harbour seal CCs that incorporated the effects of endogenously produced FAs. We do, however, note that the FA profiles of the juvenile seals and those of newly weaned seals after $>42 \mathrm{~d}$ of pure herring were easily discernible using DFA (Fig. 3) despite consuming the same herring prey. This suggests either the FA requirements of seals in early development differ from those in later life stages or that extended feeding periods in the order of months must be undertaken to ensure calibration samples correctly account for biosynthesis. Insufficient captive studies have been undertaken to assess whether the ratio of FA in the predator compared with those in the prey fed would vary with the type of species fed (Meyers 2007) or due to intrinsic factors such as physiological and reproductive status. While not the aim of this study, such an assessment seems worthwhile.

The newly developed Reduced subset (Table 4) was the suite of FA that provided the lowest misclassification rates overall and with which subsequent QFASA diet estimates were most similar to known intake (Fig. 5). The published Dietary subset (Table 4) routinely performed poorly when estimating diets largely composed of herring and also when estimating the high proportion of smelt at Biopsy C for the Smelt Only group (Fig. 5). The absence of $18: 1 \mathrm{n}-9$ as well as 18:1n-7 from the subset, among the most common and variable FA detected in the prey, may have been a particular handicap when discerning prey items. The more inclusive Extended Dietary subset performed as well as the Reduced FA subset when modelling biopsies from seals that recently consumed herring (e.g. Herring Only A, B and C and Smelt-Herring C). However, it poorly estimated diets consisting of smelt, generating significantly more false positive estimates than did the Reduced subset for the Smelt Only B, Smelt-Herring B and the Smelt Only C samples (Fig. 5). Although 15 subsets were evaluated in total (Table 4, Appendix), the possibility remains for different combinations of FA to provide fewer false positives and more accurate diet estimates. Additional optimization testing and validation of FA combinations for use with the QFASA technique should be continued in future studies. Given our results and those of others (Budge et al. 2006), we advise researchers working on alternate marine mammal species to explore the sensitivity of QFASA estimates using a range of CC sets and FA subsets.

\section{QFASA model evaluation post optimization}

QFASA diet estimates generally reflected changes in the proportion of prey fed over the course of the study for the 3 treatment groups (Fig. 6). Diet estimates within treatment groups changed significantly across sequential biopsy samples when diets were changed (Table 5). Conversely, QFASA diet estimates did not alter over time with a constant herring diet. The QFASA diet estimates also differed appropriately across diet treatments (Table 5). Excluding select prey species (e.g. coho salmon) from the prey library affected the reported contribution of other prey species, particularly those species with a relatively high degree of overlap in their FA signatures. Also, while the homogenate initially fed to all seals ( 21 to $52 \mathrm{~d}$ ) was composed of herring and salmon, no coho salmon was estimated by QFASA at Biopsy A and was only mis- 
classified in subsequent biopsies of seals eating smelt. On average, the QFASA results demonstrated that blubber FA profiles changed in a predictable manner as new prey species were consumed during a single species diet switch after the appropriate CCs and FA subsets were applied.

In our study, herring was generally the prey most reliably modelled despite some minor misclassification with capelin in 6 seals. Although herring and capelin separated in our multivariate analyses, they appeared to be less easily separated following integration into blubber. It is also not known whether our harbour seal CC values, which are based on a pure herring diet, might predispose the model to estimate a herringbased diet more dependably or whether FA modification in blubber is more complicated than can be accounted for by these relatively simple CC values. The proportion of smelt estimated in the diet was clearly affected by the inclusion of coho salmon, and to a lesser degree sandlance, in 11 of 13 seals consuming smelt. Consequently, the utmost care is required when evaluating appropriate prey libraries and interpreting QFASA estimates in cases where prey signatures overlap. In particular, various species of forage fish with high dietary overlap, and subsequently similar FA signatures, may need to be grouped in QFASA model outputs.

In this study, the model appeared to be somewhat more sensitive to prey fed in small quantities in contrast to a feeding study undertaken with grey seals (reviewed in Iverson et al. 2004). Our seals consumed $<11 \%$ mean homogenate by weight 11 to $39 \mathrm{~d}$ (mean = $20 \mathrm{~d}$ ) before the experiment, yet it was detected (albeit at $\leq 5 \%$ ) at Biopsy A and Biopsy B in the seals that consumed it most recently (Smelt Only and SmeltHerring groups) (Table 1, Fig. 6). The increased sensitivity may be due to the contrasting fat content of the capelin fed to the grey seals (mean $\pm \mathrm{SD}, 1.8 \pm 0.23 \%$ ) and the homogenate fed in this study $(23.6 \pm 2.95 \%)$, as the model accounts for relative fat content before converting each species' FA signature to a wet weight diet prediction. A low fat prey consumed in limited amounts may not be detected by QFASA, while it may still identify an extremely high fat prey fed in limited amounts or even milk consumed during suckling.

The variance analysis indicated QFASA estimates using only the known prey fed could approximate known diet proportions for individuals on pure diets at Biopsy C, but deviance was higher for the SmeltHerring group when switched from smelt back to herring (Fig. 7). Deviance between QFASA diet estimates and back-calculated recorded diets improved as more time was included prior to the biopsy. The best fit at Biopsy $\mathrm{C}$ was achieved using $65 \mathrm{~d}$ of previous diet data, a time period that was not possible at Biopsy B as most seals had not been in the rescue program for that amount of time. Very strong agreement was observed for the Herring Only seals regardless of the time frame reviewed (deviance $=0$ ) since their diet had not changed over the period reviewed. Deviance was minimal $(<9 \%)$ for individuals in the Smelt Only group whereas the individuals in the Smelt-Herring group differed by as much as $43 \%$ (mean $=24 \%$ ). Deviance in this group was due to an over-estimation of herring in the model, when compared with recorded diet, following the animal's switch back to a lipid-rich herring diet (Fig. 6). Our results suggest the new herring lipids were more readily incorporated into the blubber at the expense of the previous smelt FA signature (i.e. less smelt than expected at Biopsy $\mathrm{C}$ based on prey mass contribution following post hoc correction for fat content). Increases in body mass for the SmeltHerring seals were also skewed towards the period when herring was consumed (Table 1). Overall, these findings suggest the deviances can be explained by an increase in lipid content of the prey consumed and by associated mass gains. These results support those of the turnover analysis, wherein the process of lipid deposition and mobilization does not appear to be strictly linear.

\section{Blubber FA signature turnover}

Turnover of a FA signature in the blubber was estimated using 2 separate methods. Combining results from both approaches suggested FA turnover occurred in $\sim 1.5$ to 3 mo. The QFASA model estimated a $77 \%$ increase in smelt over $42 \mathrm{~d}$, which projected to a complete turnover of smelt after a minimum of $55 \mathrm{~d}$ (Fig. 8). This was very similar to the results obtained from variance and chi-square analyses for the Smelt Only group (Fig. 7, Table 6), which suggested 50 to $65 \mathrm{~d}$ were required for smelt turnover to occur. Back-calculating diet proportions and comparing them with QFASA estimates via chi-square statistics produced varying turnover estimates for homogenate, herring and smelt, which again suggested that different prey FA signatures may turnover at different rates. It appeared FA turnover was not a strictly linear process as the increase in the proportion of smelt detected slowed in the final $3 \mathrm{wk}$ for the Smelt Only group. If turnover does indeed slow down, then our outer estimate of 3 mo may be more realistic. It may be particularly unrealistic to assume a steady turnover in seals experiencing rapid growth as was the case in this study.

The predicted turnover rate in this study was somewhat faster than previously estimated by Kirsch et al. (2000) (25 to $50 \%$ FA turnover in 30 d) for captive harp seals Phoca groenlandica. The discrepancy could be 
attributed to the rapid growth of the harbour seals, as the seals examined by Kirsch et al. (2000) lost substantial body fat over their experiment while the seals in this study were clearly fattening. Dietary FA tend to be stored directly in adipose tissue during conditions of rapid fattening (Nelson 1992). In addition, the leaner Atlantic pollock Pollachius virens $(1.7 \pm 0.21 \%$ lipid $)$ fed to the harp seals in the Kirsch et al. (2000) study may have taken longer to be incorporated into the blubber than the smelt $(2.8 \pm 1.59 \%$ lipid $)$ consumed by the harbour seals in this study, which in turn would have generated a longer turnover prediction. This is in agreement with a similar QFASA study using captive Steller sea lions Eumetopias jubatus, in which preliminary results demonstrated turnover rate can be affected by the fat content of the prey consumed (Tollit et al. unpubl. data). As stated earlier, turnover could also be affected by the quantity of prey consumed as seals that consumed more smelt on average (Smelt Only group, $2.62 \mathrm{~kg} \mathrm{~d}^{-1}$ ) experienced a faster turnover $(63 \%)$ than did seals that consumed less (Smelt-Herring group, $1.63 \mathrm{~kg} \mathrm{~d}^{-1}, 41 \%$ ) over the same time period (Table 6, Fig. 8).

\section{Study limitations}

The arrival of the seals at the rescue centre, as well as the time required to wean them, was irregular and not under our control. The variability observed in the amount of herring $(p<0.02)$ at Biopsy A for the different groups appeared to be related to the number of days fed solid herring prior to the start of the experiment (i.e. since weaning). For example, the Herring Only group was composed of individuals that had spent a longer period of time in the rescue program (Table 1) and those seals could be distinguished from other seals that were weaned later (Table 5). While still indicative of dietary induced differences, the results indicated the treatment groups did not start the experiment with a statistically similar FA signature as was intended. A different pattern emerged after 2 treatment groups were switched to the smelt diet and then could not be distinguished from each other (Biopsy B, Table 5), but the Herring Only group, which continued to consume herring, was significantly different from these 2 groups. Therefore, it is clear that the separation of the treatment groups at Biopsy B and Biopsy C was not due to some inherent difference among the groups at Biopsy A, but rather due to subsequent dietary changes.

The incorrect detection of smelt at Biopsy A in some cases was somewhat confounding to our analyses. The false positive was possibly derived from a signature developed before the seals' arrival at the rescue centre and may have even reflected the mothers' past diet. Two seals (Pv03-12 and Pv03-20), whose diets were controlled for only 22 and $31 \mathrm{~d}$ prior to the first biopsy, respectively, were responsible for the incorrect smelt prediction in the Smelt Only group. Similarly, 3 seals (Pv03-15, Pv03-18 and Pv03-19) contributed to the error in the Smelt-Herring group when the 3 prey species library was used. Their diets were known for as few as 21 to $24 \mathrm{~d}$ before the first biopsy. If the turnover time suggested in this experiment was correct, then the limited time prior to the first biopsy, particularly when coupled with a limited prey library, could account for the high proportion of smelt detected in these individual seals before smelt were knowingly fed. This explanation has merit given that these initially high estimated smelt proportions were reduced or eliminated entirely when the 11 and 10 prey species libraries were used with the model in lieu of the 3 species library.

Seals were transferred to a large common pool specific to their diet group as they grew. While care was taken to feed the seals evenly, some individual seals undoubtedly consumed more than others. This may account for some of the variability in the diet estimates for specific seals within a treatment group, particularly the Smelt-Herring group (Fig. 7). A cross-comparison of the animal care records with model diet estimates revealed the 2 seals (Pv03-03 and Pv03-10) (Table 1) that gained the least mass over the first $21 \mathrm{~d}$ were the only individuals with smelt estimates radically below the mean for the Smelt-Herring group at Biopsy B. Their limited estimated intake combined with their limited mass gain suggested there were differences in the amount of smelt consumed by the group. However, it also suggested that variations in intake could be detected by the model.

The smelt were modelled as 3 separate size-maturity classes based on differences in their FA signatures. Despite providing the model with 3 opportunities to select smelt, the QFASA model was able to incorporate the variability in the smelt classes and provide a more accurate diet prediction than when the prey was averaged and modelled as a single group. Model estimates for specific smelt classes were re-assigned to other smelt classes when they were removed, along with coho salmon, from the prey library, suggesting the various smelt signatures were more similar to each other than to most other species. The ability of QFASA to detect differences within prey species were consistent with the findings of Iverson et al. $(1997,2002)$ and Budge et al. (2002) where size-age classes of forage fish could be characterized by their FA composition. These results may be relevant to others working with FA who may have different size, age or sex classes nested within their prey databases. 


\section{CONCLUSIONS}

The QFASA estimates correctly tracked major diet trends in the simple feeding study when fine-tuned model parameters were used despite some misclassification of similar prey species. Multivariate analyses split treatment groups reliably with as few as 6 FA and the same treatment groups were discerned statistically with results generated by the QFASA model after Biopsy A. Herring was the most reliably modelled prey compared with recorded intake, which may be the result of using calibrations based on that species. Calibrations from juvenile harbour seals were more reliable than CCs from grey seal pups, irrespective of the FA subset employed. Further testing with calibrations generated from alternate prey and under different intrinsic conditions, as well as estimating CCs via radiolabelled FA (Cooper et al. 2006), should continue. Using the newly developed Reduced FA subset provided diet estimates with the fewest false positives overall and using different FA subsets affected reported diet composition. The sensitivity of QFASA diet estimates based on free-ranging samples should be explored with a range of FA subsets, including the Reduced subset, as an initial starting point. Quantitative diet estimates were influenced by the prey species included in the model and certain prey species were consistently misclassified, particularly those identified as having similar FA signatures. Identifying problematic species and then removing them from the model produced diet estimates that were similar to known intake. Clearly this approach would be impractical for free-ranging diet studies. Therefore, previewing the prey library with DFA-like techniques and then either removing select species or grouping similar prey (e.g. forage fish) could reduce uncertainties when modelling wild samples. Alternatively, modeling a sub-sample of each prey species against the remaining prey library (i.e. as if the prey were blubber sample) would be a useful exercise to assess potential sources of overlap among prey species (Budge et al. 2006). Complete turnover of the FA signature within the blubber was clearly $>42 \mathrm{~d}$ and probably $>55 \mathrm{~d}$. Turnover may not be a strictly linear process, and may be dependent on food intake, food composition and growth patterns, but this requires further investigation. Although sensitive to the $\mathrm{CC}$ and FA subsets used, QFASA demonstrated the ability to detect modifications of FA blubber signatures after a long term and consistent switch in diet. Clearly care is required when selecting $\mathrm{CC}$ sets and FA subsets when studying pinniped diets in more complex conditions, particularly when modelling prey species with similar FA signatures or with signatures that become similar during synthesis.
Acknowledgements. We thank the staff of the Vancouver Aquarium Marine Science Centre for access to and care of the study subjects. Veterinarian Dr. D. Huff performed the biopsy procedures, S. Heaslip assisted with biopsy sampling and R. Joy assisted with figure preparation. M. J. Walton and R. Purves provided custom software. We also thank D. A. S. Rosen and 5 anonymous referees for valuable comments on earlier drafts of the manuscript. This work was conducted in accordance with the principles of the Canadian Council on Animal Care under permit no. A03-0176. Funding for this project was provided to the North Pacific Universities Marine Mammal Research Consortium by the National Oceanographic and Atmospheric Administration and the North Pacific Marine Science Foundation. Additional support was provided by research and equipments grants to S.J.I. from the Natural Sciences and Engineering Research Council (NSERC), Canada.

\section{LITERATURE CITED}

Andersen SM, Lydersen CG, Grahl-Nielsen O, Kovacs KM (2004) Autumn diet of harbour seals (Phoca vitulina) at Prins Karls Forland, Svalbard, assessed via scat and fatty acid analyses. Can J Zool 82:1230-1245

Arnould JPY, Nelson MM, Nichols PD, Oosthhuizen WH (2005) Variation in the fatty acid composition of blubber in Cape fur seals (Arctocephalus pusillus pusillus) and the implications for dietary interpretation. J Comp Physiol B Biochem Syst Environ Physiol 175:285-295

Beck CA, Iverson SJ, Bowen WD, Blanchard W (2007) Sex differences in grey seal diet reflect seasonal variation in foraging behaviour and reproductive expenditure: evidence from quantitative fatty acid signature analysis. J Anim Ecol 76:490-502

Bradshaw CJA, Hindell MA, Best NJ, Phillips KL, Wilson G, Nichols PD (2003) You are what you eat: describing the foraging ecology of southern elephant seals (Mirounga leonina) using blubber fatty acids. Proc R Soc Lond B Biol Sci 270:1283-1292

Browne P, Laake JL, DeLong RL (2002) Improving pinniped diet analyses through identification of multiple skeletal structures in fecal samples. Fish Bull 100:423-433

Budge SM, Iverson SJ, Bowen WD, Ackman RG (2002) Among- and within-species variabilty in fatty acid signatures of marine fish and invertebrates on the Scotian Shelf, Georges Bank, and southern Gulf of St. Lawrence. Can J Fish Aquat Sci 59:886-898

Budge SM, Iverson SJ, Koopman HN (2006) Studying trophic ecology in marine ecosystems using fatty acids: a primer on analysis and interpretation. Mar Mamm Sci 22:759-801

Colby RH, Mattacks CA, Pond CM (1993) The gross anatomy, cellular structure and fatty acid composition of adipose tissue in captive polar bears (Ursus maritimus). Zoo Biol 12: $267-275$

Cooper MH, Iverson SJ, Rouvinen-Watt K (2006) Metabolism of dietary cetoleic acid (22:1n-11) in mink (Mustela vison) and gray seals (Halichoerus grypus) studied using radiolabeled fatty acids. Physiol Biochem Zool 79:820-829

Dahl TM, Lydersen CG, Kovacs KM, Falk-Petersen S, Sargent J, Gjertz I, Gulliksen B (2000) Fatty acid composition of the blubber in white whales (Delphinapterus leucas). Polar Biol 23:401-409

> Folch J, Lees M, Sloane-Stanly GH (1957) A simple method for the isolation and purification of total lipids from animal tissues. J Biol Chem 226:497-509

Grahl-Nielsen O, Andersen M, Derocher AE, Lydersen C, 
Wiig $\varnothing$, Kovacs KM (2003) Fatty acid composition of the adipose tissue of polar bears and of their prey: ringed seals, bearded seals and harp seals. Mar Ecol Prog Ser 265: 275-282

Grahl-Nielsen O, Halvorsen AK, Bodoev N, Averina L and others (2005) Fatty acid composition of blubber of the Baikal seal Phoca sibirica and its marine relative, the ringed seal P. hispida. Mar Ecol Prog Ser 305:261-274

Herman DP, Burrows DG, Wade PR, Durban JW and others (2005) Feeding ecology of eastern North Pacific killer whales Orcinus orca from fatty acid, stable isotope, and organochlorine analyses of blubber biopsies. Mar Ecol Prog Ser 302:275-291

Hoberecht L (2006) Investigating the use of blubber fatty acids to detect Steller sea lion (Eumetopias jubatus) foraging on ephemeral high-quality prey. PhD thesis, University of Washington, Seattle, WA

Hooker SK, Iverson SJ, Ostrom P, Smith SC (2001) Diet of northern bottlenose whales inferred from fatty-acid and stable-isotope analyses of biopsy samples. Can J Zool 79: $1442-1454$

Iverson SJ (1993) Milk secretion in marine mammals in relation to foraging: Can milk fatty acids predict diet? Symp Zool Soc Lond 66:263-291

> Iverson SJ, Frost KJ, Lowry LF (1997) Fatty acid signatures reveal fine scale structure of foraging distribution of harbor seals and their prey in Prince William Sound, Alaska. Mar Ecol Prog Ser 151:255-271

Iverson SJ, Lang SLC, Cooper M (2001) Comparison of the Bligh and Dyer and Folch methods for total lipid determination in a broad range of marine tissues. Lipids 36: $1283-1287$

Iverson SJ, Frost KJ, Lang SLC (2002) Fat content and fatty acid composition of forage fish and invertebrates in Prince William Sound, Alaska: factors contributing to among and within species variability. Mar Ecol Prog Ser 241:161-181

Iverson SJ, Field C, Bowen WD, Blanchard W (2004) Quantitative fatty acid signature analysis: a new method of estimating predator diets. Ecol Monogr 74:211-235

Iverson SJ, Stirling I, Lang SLC (2006) Spatial and temporal variation in the diets of polar bears across the Canadian arctic: indicators of changes in prey populations and environment. Symp Zool Soc Lond Conserv Biol Ser 12:98-117

Iverson SJ, Springer AM, Kitaysky AS (2007) Seabirds as indicators of food web structure and ecosystem variability: qualitative and quantitative diet analyses using fatty acids. Mar Ecol Prog Ser 352:235-244

Jobling M, Breiby A (1986) The use and abuse of fish otoliths in studies of feeding habits of marine piscivores. Sarsia 71: 265-274

Kirsch PE, Iverson SJ, Bowen WD (2000) Effect of a low-fat diet on body composition and blubber fatty acids of captive juvenile harp seals (Phoca groenlandica). Physiol Biochem Zool 73:45-59

Laake JL, Browne P, DeLong RL (2002) Pinniped diet composition: a comparison of estimation models. Fish Bull 100: 434-447

Lea MA, Cherel Y, Guinet C, Nichols PD (2002) Antarctic fur seals foraging in the Polar Frontal Zone: inter-annual shifts in diet as shown from fecal and fatty acid analyses. Mar Ecol Prog Ser 245:281-297
Meyers AM (2007) Evaluating the fatty acid signature technique for studies of diet composition in piscivorous waterbirds. MS thesis, Oregon State University, Corvallis, OR

Nelson GJ (1992) Dietary fatty acids and lipid metabolism. In: Chow CK (ed) Fatty acids in foods and their health implications. Marcel Dekker, New York, p 437-471

Olesiuk PF, Bigg MA, Ellis GM, Crockford SJ, Wigen RJ (1990) An assessment of the feeding habits of harbour seals (Phoca vitulina) in the Strait of Georgia, British Columbia, based on scat analysis. Can Tech Rep Fish Aquat Sci 1730

Pierce GJ, Boyle PR (1991) A review of methods for diet analysis in piscivorous marine mammals. Oceanogr Mar Biol 29: 409-486

Raclot T, Groscolas R, Cherel Y (1998) Fatty acid evidence for the importance of myctophid fishes in the diet of king penguins, Aptenodytes patagonicus. Mar Biol 132:523-533

Rouvinen K, Kiiskinen T (1989) Influence of dietary fat source on the body fat composition of mink (Mustela vison) and blue fox (Alopex lagapus). Acta Agric Scand Sect A Anim Sci 39:279-288

Ruchonnet D, Boutoute M, Guinet C, Mayzaud P (2006) Fatty acid composition of Mediterranean fin whale Balaenoptera physalus blubber with respect to body heterogeneity and trophic interation. Mar Ecol Prog Ser 311: 165-174

Samuel AM, Worthy GAJ (2004) Variability in fatty acid composition of bottlenose dolphin (Tursiops truncatus) blubber as a function of body site, season, and reproductive state. Can J Zool 82:1933-1942

Smith RJ, Hobson KA, Koopman HN, Lavigne DM (1996) Distinguishing between populations of fresh- and salt-water harbour seals (Phoca vitulina) using stable-isotope ratios and fatty acid profiles. Can J Fish Aquat Sci 53:272-279

Staniland IJ (2002) Investigating the biases in the use of hard prey remains to identify diet composition using Antarctic fur seals (Arctocephalus gazella) in captive feeding trials. Mar Mamm Sci 18:223-243

> Thiemann GW, Budge SM, Iverson SJ (2004) Determining blubber fatty acid composition: a comparison of in situ direct and traditional methods. Mar Mamm Sci 20: 284-295

Thiemann GW, Iverson SJ, Stirling I (2006) Seasonal, sexual and anatomical variability in the adipose tissue of polar bears (Ursus maritimus). J Zool 269:65-76

Tollit DJ, Wong M, Winship AJ, Rosen DAS, Trites AW (2003) Quantifying errors associated with using prey skeletal structures from fecal samples to determine the diet of Steller's sea lion (Eumetopias jubatus). Mar Mamm Sci 19: 724-744

Tollit DJ, Heaslip S, Deagle B, Iverson SJ, Joy R, Rosen DAS, Trites AW (2006) Estimating diet composition in sea lions: which technique to choose? In: Trites A, Atkinson S, DeMaster D, Fritz L, Gelatt T, Rea L, Whynne K (eds) Sea lions of the world. Alaska Sea Grant College Program, University of Alaska, Fairbanks, AK, p 293-308

Trites AW, Joy R (2005) Dietary analysis from fecal samples: How many scats are enough? J Mammal 86:704-712

Walton M, Pomeroy P (2003) Use of blubber fatty acid profiles to detect inter-annual variations in the diet of grey seals Halichoerus grypus. Mar Ecol Prog Ser 248:257-266

Zar J (1996) Biostatistical analyses. Prentice Hall, Upper Saddle River, NJ 
Appendix 1. Phoca vitulina. Additional fatty acid subsets tested using 3 biopsies from 6 harbour seals ( 2 seals from each treatment group, $\mathrm{N}=18$ biopsies total)

\begin{tabular}{|c|c|c|c|c|c|c|c|c|c|c|c|c|}
\hline Fatty acid & Test 1 & Test 2 & Test 3 & Test 4 & Test 5 & Test 6 & Test 7 & Test 8 & Test 9 & Test 10 & Test 11 & Test 12 \\
\hline $14: 0$ & $X$ & & & & & & & & & & & \\
\hline $16: 0$ & $\mathrm{X}$ & & $\mathrm{X}$ & & & & & & $\mathrm{X}$ & $\mathrm{X}$ & $\mathrm{X}$ & \\
\hline $16: 1 n-11$ & $\mathrm{X}$ & & & & & & & & & & & \\
\hline 16:1n-9 & $\mathrm{X}$ & & & & & & & & & & & \\
\hline $16: 1 n-7$ & $\mathrm{X}$ & & & & $\mathrm{X}$ & & & & $\mathrm{X}$ & $\mathrm{X}$ & & $\mathrm{X}$ \\
\hline $16: 2 n-6$ & $\mathrm{X}$ & $\mathrm{X}$ & $\mathrm{X}$ & & $\mathrm{X}$ & $\mathrm{X}$ & $\mathrm{X}$ & $\mathrm{X}$ & $\mathrm{X}$ & $\mathrm{X}$ & $\mathrm{X}$ & $\mathrm{X}$ \\
\hline $16: 2 n-4$ & $\mathrm{X}$ & $\mathrm{X}$ & $\mathrm{X}$ & $\mathrm{X}$ & $\mathrm{X}$ & $\mathrm{X}$ & $\mathrm{X}$ & $\mathrm{X}$ & $\mathrm{X}$ & $\mathrm{X}$ & $\mathrm{X}$ & $\mathrm{X}$ \\
\hline $16: 3 n-6$ & $\mathrm{X}$ & $\mathrm{X}$ & $\mathrm{X}$ & $\mathrm{X}$ & $\mathrm{X}$ & $\mathrm{X}$ & $\mathrm{X}$ & $\mathrm{X}$ & $\mathrm{X}$ & $\mathrm{X}$ & $\mathrm{X}$ & $\mathrm{X}$ \\
\hline $16: 3 n-4$ & & $\mathrm{X}$ & $\mathrm{X}$ & $\mathrm{X}$ & $\mathrm{X}$ & $\mathrm{X}$ & $\mathrm{X}$ & $\mathrm{X}$ & $\mathrm{X}$ & $\mathrm{X}$ & $\mathrm{X}$ & $\mathrm{X}$ \\
\hline $16: 3 n-1$ & & $\mathrm{X}$ & $\mathrm{X}$ & $\mathrm{X}$ & $\mathrm{X}$ & $\mathrm{X}$ & $\mathrm{X}$ & $\mathrm{X}$ & $\mathrm{X}$ & $\mathrm{X}$ & $\mathrm{X}$ & $\mathrm{X}$ \\
\hline $16: 4 n-3$ & $\mathrm{X}$ & $\mathrm{X}$ & $\mathrm{X}$ & $\mathrm{X}$ & $\mathrm{X}$ & $\mathrm{X}$ & $\mathrm{X}$ & $\mathrm{X}$ & $\mathrm{X}$ & $\mathrm{X}$ & $\mathrm{X}$ & $\mathrm{X}$ \\
\hline $16: 4 n-1$ & $X$ & $\mathrm{X}$ & $\mathrm{X}$ & $\mathrm{X}$ & $\mathrm{X}$ & $\mathrm{X}$ & $\mathrm{X}$ & $\mathrm{X}$ & $\mathrm{X}$ & $\mathrm{X}$ & $\mathrm{X}$ & $\mathrm{X}$ \\
\hline $18: 0$ & $X$ & & & & & & & & & & & \\
\hline $18: 1 n-9$ & $\mathrm{X}$ & & $\mathrm{X}$ & $\mathrm{X}$ & $\mathrm{X}$ & $\mathrm{X}$ & $\mathrm{X}$ & $\mathrm{X}$ & $\mathrm{X}$ & $\mathrm{X}$ & $\mathrm{X}$ & $\mathrm{X}$ \\
\hline $18: 1 n-7$ & $\mathrm{X}$ & $\mathrm{X}$ & $\mathrm{X}$ & $\mathrm{X}$ & $\mathrm{X}$ & $\mathrm{X}$ & $\mathrm{X}$ & $\mathrm{X}$ & $\mathrm{X}$ & $\mathrm{X}$ & $\mathrm{X}$ & $\mathrm{X}$ \\
\hline $18: 1 n-5$ & $\mathrm{X}$ & & & & & & & & & & & \\
\hline $18: 2 n-6$ & $\mathrm{X}$ & $\mathrm{X}$ & $\mathrm{X}$ & $\mathrm{X}$ & $\mathrm{X}$ & $\mathrm{X}$ & $\mathrm{X}$ & $\mathrm{X}$ & $\mathrm{X}$ & $\mathrm{X}$ & $\mathrm{X}$ & $\mathrm{X}$ \\
\hline $18: 2 n-4$ & $\mathrm{X}$ & $X$ & $\mathrm{X}$ & $\mathrm{X}$ & $\mathrm{X}$ & $\mathrm{X}$ & $\mathrm{X}$ & $\mathrm{X}$ & $\mathrm{X}$ & $\mathrm{X}$ & $\mathrm{X}$ & $\mathrm{X}$ \\
\hline $18: 3 n-6$ & $\mathrm{X}$ & $\mathrm{X}$ & $\mathrm{X}$ & $\mathrm{X}$ & $\mathrm{X}$ & $\mathrm{X}$ & $\mathrm{X}$ & $\mathrm{X}$ & $\mathrm{X}$ & $\mathrm{X}$ & $\mathrm{X}$ & $\mathrm{X}$ \\
\hline $18: 3 n-4$ & $\mathrm{X}$ & $\mathrm{X}$ & $\mathrm{X}$ & $\mathrm{X}$ & $\mathrm{X}$ & $\mathrm{X}$ & $\mathrm{X}$ & $\mathrm{X}$ & $\mathrm{X}$ & $\mathrm{X}$ & $\mathrm{X}$ & $\mathrm{X}$ \\
\hline $18: 3 n-3$ & $\mathrm{X}$ & $\mathrm{X}$ & $\mathrm{X}$ & $\mathrm{X}$ & $\mathrm{X}$ & $\mathrm{X}$ & $\mathrm{X}$ & $\mathrm{X}$ & $\mathrm{X}$ & $\mathrm{X}$ & $\mathrm{X}$ & $\mathrm{X}$ \\
\hline $18: 3 n-1$ & & $\mathrm{X}$ & $\mathrm{X}$ & $\mathrm{X}$ & $\mathrm{X}$ & $\mathrm{X}$ & $\mathrm{X}$ & $\mathrm{X}$ & $\mathrm{X}$ & $\mathrm{X}$ & $\mathrm{X}$ & $\mathrm{X}$ \\
\hline $18: 4 n-3$ & $\mathrm{X}$ & $\mathrm{X}$ & $\mathrm{X}$ & $\mathrm{X}$ & $\mathrm{X}$ & $\mathrm{X}$ & $\mathrm{X}$ & $\mathrm{X}$ & $\mathrm{X}$ & $\mathrm{X}$ & $\mathrm{X}$ & $\mathrm{X}$ \\
\hline $18: 4 n-1$ & $\mathrm{X}$ & $\mathrm{X}$ & $\mathrm{X}$ & $\mathrm{X}$ & $\mathrm{X}$ & $\mathrm{X}$ & $\mathrm{X}$ & $\mathrm{X}$ & $\mathrm{X}$ & $\mathrm{X}$ & $\mathrm{X}$ & $\mathrm{X}$ \\
\hline 20:1n-11 & $\mathrm{X}$ & $\mathrm{X}$ & $\mathrm{X}$ & $\mathrm{X}$ & $\mathrm{X}$ & & $\mathrm{X}$ & $\mathrm{X}$ & & & & \\
\hline $20: 1 n-9$ & $\mathrm{X}$ & $X$ & $\mathrm{X}$ & $\mathrm{X}$ & $\mathrm{X}$ & $\mathrm{X}$ & $\mathrm{X}$ & $\mathrm{X}$ & $\mathrm{X}$ & $\mathrm{X}$ & $\mathrm{X}$ & $\mathrm{X}$ \\
\hline $20: 1 n-7$ & $\mathrm{X}$ & $X$ & $\mathrm{X}$ & $\mathrm{X}$ & $\mathrm{X}$ & $\mathrm{X}$ & $\mathrm{X}$ & $\mathrm{X}$ & $\mathrm{X}$ & $\mathrm{X}$ & $\mathrm{X}$ & $\mathrm{X}$ \\
\hline $20: 2 n-6$ & $\mathrm{X}$ & $X$ & $\mathrm{X}$ & $\mathrm{X}$ & $\mathrm{X}$ & $\mathrm{X}$ & $\mathrm{X}$ & $\mathrm{X}$ & $\mathrm{X}$ & $\mathrm{X}$ & $\mathrm{X}$ & $\mathrm{X}$ \\
\hline $20: 3 n-6$ & $\mathrm{X}$ & $\mathrm{X}$ & $\mathrm{X}$ & $\mathrm{X}$ & $\mathrm{X}$ & $\mathrm{X}$ & & $\mathrm{X}$ & $\mathrm{X}$ & $\mathrm{X}$ & $\mathrm{X}$ & $\mathrm{X}$ \\
\hline $20: 4 n-6$ & $\mathrm{X}$ & $X$ & $\mathrm{X}$ & $\mathrm{X}$ & $\mathrm{X}$ & $\mathrm{X}$ & $\mathrm{X}$ & $\mathrm{X}$ & $\mathrm{X}$ & $\mathrm{X}$ & $\mathrm{X}$ & $\mathrm{X}$ \\
\hline $20: 3 n-3$ & $\mathrm{X}$ & $\mathrm{X}$ & $\mathrm{X}$ & $\mathrm{X}$ & $\mathrm{X}$ & $\mathrm{X}$ & $\mathrm{X}$ & $\mathrm{X}$ & $\mathrm{X}$ & $\mathrm{X}$ & $\mathrm{X}$ & $\mathrm{X}$ \\
\hline $20: 4 n-3$ & $\mathrm{X}$ & $\mathrm{X}$ & $\mathrm{X}$ & $\mathrm{X}$ & $\mathrm{X}$ & $\mathrm{X}$ & $\mathrm{X}$ & $\mathrm{X}$ & $\mathrm{X}$ & $\mathrm{X}$ & $\mathrm{X}$ & $\mathrm{X}$ \\
\hline $20: 5 n-3$ & $\mathrm{X}$ & $X$ & $\mathrm{X}$ & $\mathrm{X}$ & $\mathrm{X}$ & $\mathrm{X}$ & $\mathrm{X}$ & $\mathrm{X}$ & $\mathrm{X}$ & $\mathrm{X}$ & $\mathrm{X}$ & $\mathrm{X}$ \\
\hline $22: 1 n-11$ & $\mathrm{X}$ & $\mathrm{X}$ & $\mathrm{X}$ & $\mathrm{X}$ & $\mathrm{X}$ & $\mathrm{X}$ & $\mathrm{X}$ & $\mathrm{X}$ & $\mathrm{X}$ & $\mathrm{X}$ & $\mathrm{X}$ & $\mathrm{X}$ \\
\hline $22: 1 n-9$ & $\mathrm{X}$ & $\mathrm{X}$ & $\mathrm{X}$ & $\mathrm{X}$ & $\mathrm{X}$ & $\mathrm{X}$ & $\mathrm{X}$ & $\mathrm{X}$ & $\mathrm{X}$ & $\mathrm{X}$ & $\mathrm{X}$ & $\mathrm{X}$ \\
\hline $22: 1 n-7$ & $\mathrm{X}$ & $\mathrm{X}$ & $\mathrm{X}$ & $\mathrm{X}$ & $\mathrm{X}$ & $\mathrm{X}$ & $\mathrm{X}$ & $\mathrm{X}$ & $\mathrm{X}$ & $\mathrm{X}$ & $\mathrm{X}$ & $\mathrm{X}$ \\
\hline $22: 2 n-6$ & & $\mathrm{X}$ & $\mathrm{X}$ & $\mathrm{X}$ & $\mathrm{X}$ & $\mathrm{X}$ & $\mathrm{X}$ & $\mathrm{X}$ & $\mathrm{X}$ & $\mathrm{X}$ & $\mathrm{X}$ & $\mathrm{X}$ \\
\hline $21: 5 n-3$ & $\mathrm{X}$ & $\mathrm{X}$ & $\mathrm{X}$ & $\mathrm{X}$ & $\mathrm{X}$ & $\mathrm{X}$ & $\mathrm{X}$ & $\mathrm{X}$ & $\mathrm{X}$ & $\mathrm{X}$ & $\mathrm{X}$ & $\mathrm{X}$ \\
\hline $22: 4 n-6$ & $\mathrm{X}$ & $\mathrm{X}$ & $\mathrm{X}$ & & $\mathrm{X}$ & $\mathrm{X}$ & $\mathrm{X}$ & $\mathrm{X}$ & $\mathrm{X}$ & $\mathrm{X}$ & $\mathrm{X}$ & $\mathrm{X}$ \\
\hline $22: 5 n-6$ & $\mathrm{X}$ & $\mathrm{X}$ & $\mathrm{X}$ & & $\mathrm{X}$ & & & & $\mathrm{X}$ & & & \\
\hline $22: 4 n-3$ & $\mathrm{X}$ & $\mathrm{X}$ & $\mathrm{X}$ & $\mathrm{X}$ & $\mathrm{X}$ & & & & & & & \\
\hline $22: 5 n-3$ & $\mathrm{X}$ & & & & & & & & & & & \\
\hline $22: 6 n-3$ & $\mathrm{X}$ & $\mathrm{X}$ & $\mathrm{X}$ & $\mathrm{X}$ & $\mathrm{X}$ & $\mathrm{X}$ & $\mathrm{X}$ & $\mathrm{X}$ & $\mathrm{X}$ & $\mathrm{X}$ & $\mathrm{X}$ & $\mathrm{X}$ \\
\hline
\end{tabular}

Editorial responsibility: Otto Kinne, Oldendorf/Luhe, Germany
Submitted: July 5, 2007; Accepted: December 17, 2007

Proofs received from author(s): April 29, 2008 